\title{
Guaranteed energy error bounds for the Poisson equation using a flux-free approach: solving the local problems in subdomains
}

\author{
N. Parés ${ }^{1,4}$, H. Santos ${ }^{2}$ and P. Díez ${ }^{3,4, *}$ \\ 1 Escola Universitària d'Enginueria Tècnica Industrial de Barcelona, Universitat Politècnica de Catalunya \\ 2 Departamento de Engenharia Civil, Instituto Superior Técnico, Av. Rovisco Pais, 1049-001 Lisboa, \\ Portugal \\ 3 Escola Tècnica Superior d'Enginyers de Camins, Canals i Ports, Universitat Politècnica de Catalunya \\ 4 Laboratori de Càlcul Numèric (LaCàN), Departament de Matemàtica Aplicada III, \\ C/Jordi Girona 1-3 E-08034 Barcelona Spain
}

\begin{abstract}
SUMMARY
A method to compute guaranteed upper bounds for the energy norm of the exact error in the finite element solution of the Poisson equation is presented. The bounds are guaranteed for any finite element mesh however coarse it may be, not just in the asymptotic regime. The bounds are constructed by employing a subdomain based a posteriori error estimate which yields self-equilibrated residual loads in stars (patches of elements). The proposed approach is an alternative to standard equilibrated residual methods providing sharper bounds. The use of a flux-free error estimator improves the effectivities of the upper bounds for the energy while retaining the certainty of the bounds.

KEY WORDS: Exact/guaranteed/strict bounds; Poisson equation; Error estimation; Adaptivity; Fluxfree estimate; Subdomain a posteriori error estimation; Partition of unity; Residual based estimators
\end{abstract}

\section{INTRODUCTION}

A posteriori error estimation techniques are nowadays essential tools to certify the reliability of numerical simulations in any engineering design process. In practice, it is not sufficient to assess a global measure of the error, the accuracy has to be controlled for a given quantity of interest or functional output (depending on the solution field).

Most of the currently used strategies to obtain upper and lower bounds for linear functional outputs require estimating the error in global energy-type norms. Thus, obtaining sharp bounds for the energy norm of the error is a key issue also in the goal-oriented error assessment. In particular, for the advection-diffusion equation, upper bounds for the energy norm of the error are required for an associated Poisson problem [1].

The present work aims at obtaining sharp and strict upper bounds for the error in the energy norm of finite element approximations of the Poisson equation. This is regarded as a basic tool that may be subsequently used to assess the error in linear outputs of the more general advection-reaction-diffusion equation. The approach presented here combines the fluxfree domain decomposition strategy with a dual formulation of the resulting local problems yielding a guaranteed upper bound of the solution.

\footnotetext{
*Correspondence to: P. Díez. Tel.: +34 934017240. Fax: +34 934011825. E-mail address: pedro.diez@upc.edu. URL: http://www-lacan.upc.edu.

Contract/grant sponsor: Ministerio de Educacion y Ciencia; contract/grant number: DPI2007-62395

Contract/grant sponsor: Ministerio de Educacion y Ciencia; contract/grant number: BIA2007-66965

Contract/grant sponsor: Generalitat de Catalunya AGAUR; contract/grant number: 2005SGR917
} 
The dual formulation of the problems follows the ideas introduced in the early 1970s by Fraeijs de Veubeke who proposed a methodology to obtain an upper bound for the energy norm of the error minimizing the complementary energy of a global dual approximation (flux fulfilling the balance equations) [2]. Although the resulting bounds are sharp, the global nature of the dual approximation yields a relatively costly method. A remedy for the high computational cost of these methods is to obtain a dual solution solving only local problems. In the early 1980s, Kelly [3] and Ladevèze and Leguillon [4] proposed the construction of locally equilibrated fluxes to avoid the global computation of the dual approximation. This approach is widely known as the equilibrated residual method but it has also been named after hybrid-flux residual method.

The equilibrated residual method is probably the most popular implicit residual type a posteriori error estimator. This method is based on first constructing a set of equilibrated fluxes on the edges of the finite element mesh. These fluxes are the Neumann boundary conditions for the elementary infinite-dimensional problems to recover the local approximation of the error. The solutions of these problems directly yield an upper bound on the energy norm of the error. The initial idea of [4] of solving the local elementary Neumann problems using a local complementary energy approach was immediately replaced by solving the local problems approximately using a local finite element mesh $[5,6,7,8,9,10]$. This approach has been extensively used but it does not provide any certainty on the upper bounds. The error introduced in the solution of the local problem may result in an underestimation of the error norm.

It has not been until the mid 2000s $[11,12,13,14,15,16,17]$ that the initial idea of [4] has been revisited to unambiguously certify the numerical results. It is worth noting that while a proper global dual approximation of the problem may be used to obtain sharp bounds for the
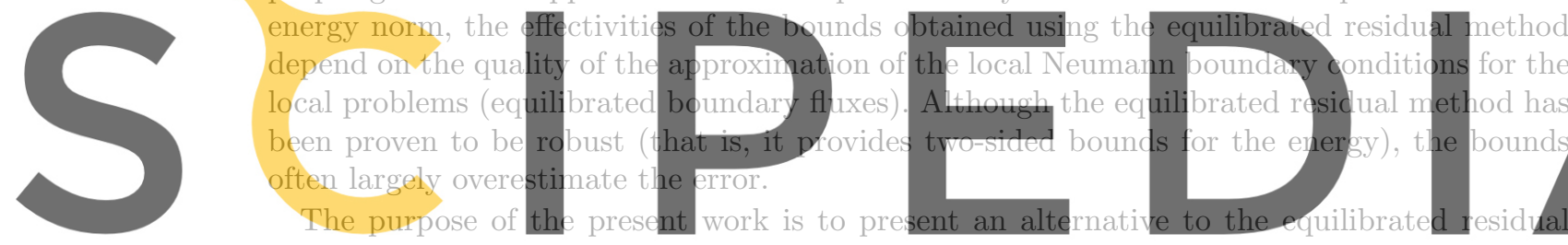

method to produce strict bounds for the energy norm of the error which better effectivities.

introduced in [18] guarantees that these local problems are directly self-equilibrated so there is no necessity to previously post-process the finite element solution to obtain equilibrated boundary fluxes. The local complementary energy approach is then used in each star to derive the strict upper bounds for the energy norm of the error.

The important issue of obtaining a lower bound is not discussed here but it may be easily obtained using the ideas in $[19,18]$. Both the upper bounds and the lower bounds for the energy norm may be then used to assess quantities of interest for selfadjoint or non-selfadjoint problems. In [1] the ideas given here have been extended to compute strict upper and lower bounds for linear quantities of interest of the advection-reaction-diffusion equation. Also, reference [20] provides the extension of this technique to linear elasticity. A similar approach is followed in [21].

The strategy presented here can also be used to recover strict bounds from the asymptotic flux-free estimate presented in [22]. This is because in this work the bilinear operator in the residual equation is split using a partition of the unity conformed by piecewise constant functions. Applying the same ideas to the estimators using non-piecewise constant partition of unity in the operator $[23,24]$ is not obvious. In any case, we follow here the ideas presented in [18] because the resulting estimates are much sharper in the asymptotic version and they are expected to keep the same behavior in the exact bound version.

The remainder of the paper is structured as follows. The model problem and the error equations are presented in the next section. The flux-free method presented in [18] is briefly described in section 3 where the idea of guaranteed and asymptotic bounds is also introduced. Section 4 discusses the complementary energy approach and its application to the flux- 
free estimate. A detailed explanation of the algorithm to numerically implement the error estimation strategy is given in sections 5 and 6 . We conclude with numerical examples illustrating the performance of the estimator, and clearly showing that the new technique provides much sharper bounds than the standard equilibrated residual method.

\section{MODEL PROBLEM AND FINITE ELEMENT APPROXIMATION}

Let $\Omega$ be an open bounded polygonal domain in $\mathbb{R}^{2}$, with boundary $\partial \Omega=\Gamma_{\mathrm{N}} \cup \Gamma_{\mathrm{D}}$, where $\Gamma_{\mathrm{N}}$ and $\Gamma_{\mathrm{D}}$ form a disjoint partition of the boundary, and $\Gamma_{\mathrm{D}}$ is a non-empty set. The boundary value problem to be solved is stated as follows: find the real-valued function $u$ such that

$$
\begin{aligned}
-\Delta u & =f & & \text { in } \Omega, \\
u & =u_{\mathrm{D}} & & \text { on } \Gamma_{\mathrm{D}}, \\
\nabla u \cdot \boldsymbol{n} & =g & & \text { on } \Gamma_{\mathrm{N}},
\end{aligned}
$$

where the Dirichlet boundary condition $u_{\mathrm{D}}$ is assumed to be continuous piecewise linear on $\Gamma_{\mathrm{D}}$.

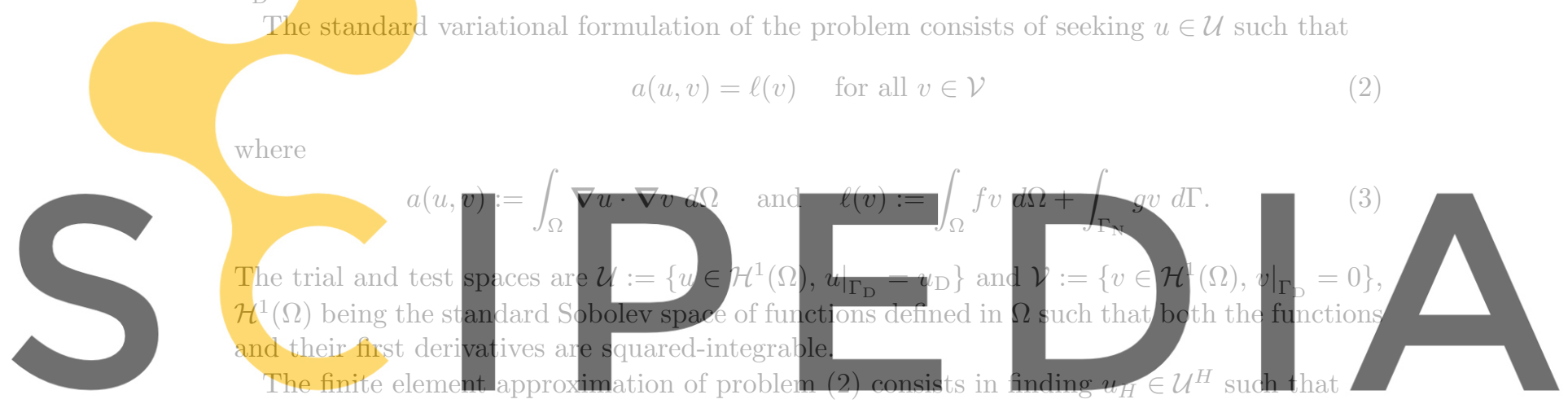

Register for free at https//www.scipedia.com to download the version without the watermark

Here, $\mathcal{U}^{H} \subset \mathcal{U}$ and $\mathcal{V}^{H} \subset \mathcal{V}$ denote the finite-dimensional spaces associated with a finite element mesh of characteristic mesh size $H$. The mesh is the union of nonoverlapping $n_{\mathrm{el}}$ linear triangular elements such that the nonempty intersection of a distinct pair of elements is either a single common node or a single common edge. For the sake of simplicity, the presentation concerns only linear elements, but the methodology is general and it is also applicable to higher-order triangular elements.

\section{FLUX-FREE ERROR ESTIMATES AND ASYMPTOTIC UPPER BOUND}

\subsection{Weak form of the residual global problem}

The purpose of the present work is to develop a method for obtaining computable strict upper bounds for the error $e:=u-u_{H}$ in the finite element approximation $u_{H}$ measured in the energy norm. That is, if $\|v\|:=a(v, v)^{1 / 2}$ denotes the energy norm of the function $v$, the goal is to obtain an upper bound of $\|e\|$.

The global equation for the error is recovered from (2) replacing the exact solution $u$ by $u_{H}+e$ and using the linearity of $a(\cdot, \cdot)$ in the first argument. In this way, the exact error $e$ lies in $\mathcal{V}$ (vanishes on $\Gamma_{\mathrm{D}}$ ) and is such that

$$
a(e, v)=\ell(v)-a\left(u_{H}, v\right)=: R(v) \quad \text { for all } v \in \mathcal{V}
$$

where $R(\cdot)$ stands for the weak residual associated with the finite element approximation $u_{H}$. 


\subsection{Domain decomposition and upper bound of the error}

Let $\phi_{i}, i=1, \ldots, n_{\mathrm{np}}$, be the set of linear shape functions associated with the $n_{\mathrm{np}}$ vertex nodes of the mesh. The support of each $\phi_{i}$ is denoted by $\omega_{i}$ and is referred to as the patch of elements connected to node $i$ of the mesh or the star associated with node $i$.

The functions $\left\{\phi_{1}, \ldots, \phi_{n_{\mathrm{np}}}\right\}$ are a partition of unity, that is

$$
\sum_{i=1}^{n_{\mathrm{np}}} \phi_{i}(\boldsymbol{x})=1 \quad \text { for all } \boldsymbol{x} \in \Omega .
$$

Using this property together with the linearity of $R(\cdot)$, (5) is rewritten as

$$
a(e, v)=R\left(v\left(\sum_{i=1}^{n_{\mathrm{np}}} \phi_{i}\right)\right)=\sum_{i=1}^{n_{\mathrm{np}}} R\left(\phi_{i} v\right) \quad \text { for all } v \in \mathcal{V} .
$$

Following [18], equation (7) is split into $n_{\mathrm{np}}$ local problems defined in every star $\omega_{i}$

$$
a_{\omega_{i}}\left(e_{\omega_{i}}, v\right)=R\left(\phi_{i} v\right) \quad \text { for all } v \in \mathcal{H}^{1}\left(\omega_{i}\right),
$$

where $a_{\omega_{i}}(\cdot, \cdot)$ is the restriction of $a(\cdot, \cdot)$ to $\omega_{i}$, namely

$$
a_{\omega_{i}}(w, v):=\int_{\omega_{i}} \nabla w \cdot \nabla v d \Omega .
$$

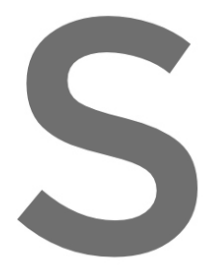

The error estimation strategy introduced in [18] consists in solving the local problems (8) and
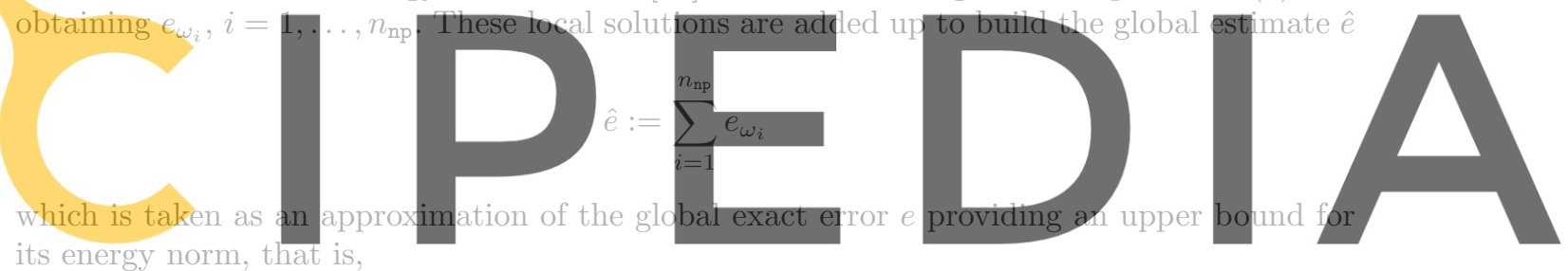

its energy norm, that is,

as stated in detail in [18], the energy norm appearing in equation (10) must be modified to allow discontinuous functions in its argument. Thus, for $v$ discontinuous along the edges of the mesh, $\|v\|^{2}$ is computed as a sum of the squared local norms, associated with the elements and, hence, integrating only in the interior of the elements.

Note that since the support of the function $\phi_{i} v$ is the star $\omega^{i}$, the weighted residual in the right-hand side (r.h.s.) term of (8) is expressed as

$$
R\left(\phi_{i} v\right)=\int_{\omega_{i}} f \phi_{i} v d \Omega+\int_{\Gamma_{\mathrm{N}} \cap \partial \omega_{i}} g \phi_{i} v d \Gamma-\int_{\omega_{i}} \nabla u_{H} \cdot \nabla\left(\phi_{i} v\right) d \Omega
$$

\subsection{Upper bound of a reference error}

The solutions of the problems (5) and (8) cannot be computed exactly because they are posed in infinite dimensional spaces, either in the global domain $\Omega$ or in a star $\omega^{i}$. Consequently, a practical alternative is required. The standard approach is to solve these problems numerically, in a reference mesh much finer than the computational mesh. This reference mesh and the corresponding approximation space are denoted using the characteristic mesh size $h$, it is assumed then that $h<<H$. Associated with this truth mesh a reference solution $u_{h}$ is introduced such that

$$
a\left(u_{h}, v\right)=\ell(v) \quad \text { for all } v \in \mathcal{V}^{h} .
$$

It is assumed that $u_{h}$ is a much better approximation to $u$ than $u_{H}$ and, consequently, that the reference error, $e_{h}:=u_{h}-u_{H}$, is a good approximation of $e$. Due to the linearity of $a(\cdot, \cdot)$ 
with respect to the first argument, the reference error can also be seen as the discrete solution of $(5)$ in $\mathcal{V}^{h}$, namely

$$
a\left(e_{h}, v\right)=R(v) \quad \text { for all } v \in \mathcal{V}^{h} .
$$

As previously said, the solution of the local problem (8) must also be approximated numerically. The standard approach is to use a finer mesh locally [18, 23, 22, 24], that is, equation (8) is solved in a discrete space which is the restriction of the reference space $\mathcal{V}^{h}$ to the star $\omega_{i}$. By doing this, the resulting local estimates $e_{\omega_{i}}^{h}$ sum up in a global estimate that provides an upper bound of the reference error, $\left\|e_{h}\right\|$, and not anymore an upper bound of the exact error, $\|e\|$. This kind of error bounds are referred as asymptotic because they are indeed bounds only for $h$ tending to zero. The strategies are said to be exact if they provide bounds of the error that can be guaranteed regardless of the mesh size used both in the computation and the error assessment.

\section{EXACT ERROR BOUNDS USING THE FLUX-FREF APPROACH}

\section{A dual formulation based on the principle of minimizing the complementary energy is used to obtain a fully computable approximation of the error that provides an upper bound of the energy norm of the exact error. This approach has been applied in the context of hybrid-flux residual estimators $[25,26]$ and it is extended in the present paper to the flux-free methodology. \\ A first step in formulating the dual solution of a problem given in its weak form, as (5) or (8), is to write it in its strong form.}
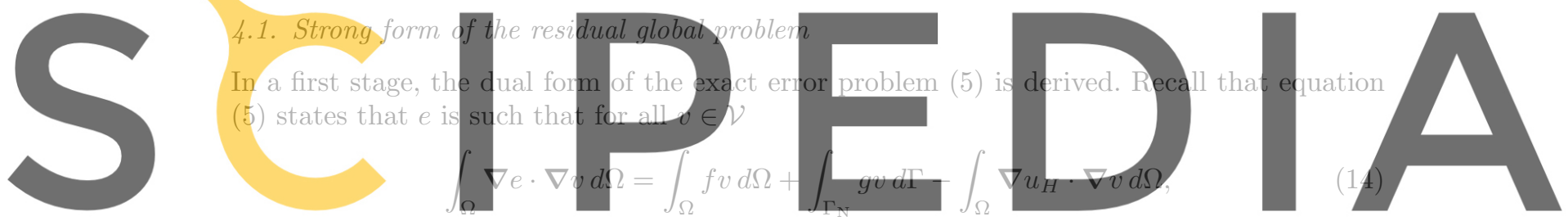

which can be rewritten as

Register for free at https//www/scipedia.com to doywlogad the version without the watermark

The left-hand side (1.h.s.) of the previous equation is rearranged integrating by parts and accounting for the fact that $\nabla e$ and $\nabla u_{H}$ are discontinuous across the element boundaries (although $\nabla e+\nabla u_{H}=\nabla u$ is continuous in $\Omega$ ):

$$
\begin{aligned}
\int_{\Omega}\left(\nabla e+\nabla u_{H}\right) \cdot \nabla v d \Omega= & \sum_{k=1}^{n_{\mathrm{el}}} \int_{\Omega_{k}}\left(\nabla e+\nabla u_{H}\right) \cdot \nabla v d \Omega \\
= & \sum_{k=1}^{n_{\mathrm{el}}}\left\{-\int_{\Omega_{k}}\left(\Delta e+\Delta u_{H}\right) v d \Omega+\sum_{\gamma \in \partial \Omega_{k}} \int_{\gamma}\left(\nabla e+\nabla u_{H}\right) \cdot \boldsymbol{n}_{k}^{\gamma} v d \Gamma\right\} \\
= & -\sum_{k=1}^{n_{\mathrm{el}}} \int_{\Omega_{k}}\left(\Delta e+\Delta u_{H}\right) v d \Omega \\
& +\sum_{\gamma \in \Gamma_{\mathrm{int}}} \int_{\gamma} \llbracket\left(\nabla e+\nabla u_{H}\right) \cdot \boldsymbol{n}^{\gamma} \rrbracket v d \Gamma+\int_{\Gamma_{N}}\left(\nabla e+\nabla u_{H}\right) \cdot \boldsymbol{n} v d \Gamma .
\end{aligned}
$$

Here $\Omega_{k}$ denotes an element of the finite element mesh and $\Gamma_{\text {int }}$ stands for the union of the interelement edges. Also, for each edge $\gamma$ a unit normal direction $\boldsymbol{n}^{\gamma}$ is assigned such that if $\gamma$ is an exterior edge $\boldsymbol{n}^{\gamma}$ coincides with the outward unit normal to $\partial \Omega, \boldsymbol{n}$. Similarly, given an element $\Omega_{k}$ and an edge of this element $\gamma \in \partial \Omega_{k}$, the outward normal to the element associated with $\gamma$ is denoted by $\boldsymbol{n}_{k}^{\gamma}$. With this notation, if $\gamma=\partial \Omega_{k} \cap \partial \Omega_{l}$,

$$
\llbracket \boldsymbol{\nabla} w \cdot \boldsymbol{n}^{\gamma} \rrbracket=\left.\nabla w\right|_{\Omega_{k}} \cdot \boldsymbol{n}_{k}^{\gamma}+\left.\nabla w\right|_{\Omega_{l}} \cdot \boldsymbol{n}_{l}^{\gamma}=\left(\left.\nabla w\right|_{\Omega_{k}}\left(\boldsymbol{n}^{\gamma} \cdot \boldsymbol{n}_{k}^{\gamma}\right)+\left.\nabla w\right|_{\Omega_{l}}\left(\boldsymbol{n}^{\gamma} \cdot \boldsymbol{n}_{l}^{\gamma}\right)\right) \cdot \boldsymbol{n}^{\gamma} .
$$


Note that, for $\gamma \in \Gamma_{\text {int }}, \boldsymbol{n}^{\gamma}$ is arbitrarily chosen to be either $\boldsymbol{n}^{\gamma}=\boldsymbol{n}_{k}^{\gamma}$ or $\boldsymbol{n}^{\gamma}=\boldsymbol{n}_{l}^{\gamma}$ and the definition of $\llbracket \nabla w \cdot \boldsymbol{n}^{\gamma} \rrbracket$ is independent of this choice.

Thus, joining equations (15) and (16) yields the following strong form:

$$
\begin{aligned}
-\left(\Delta e+\Delta u_{H}\right) & =f & & \text { in } \cup_{k} \Omega_{k}, \\
\left(\boldsymbol{\nabla} e+\nabla u_{H}\right) \cdot \boldsymbol{n} & =g & & \text { on } \Gamma_{\mathrm{N}}, \\
\llbracket\left(\boldsymbol{\nabla} e+\boldsymbol{\nabla} u_{H}\right) \cdot \boldsymbol{n}^{\gamma} \rrbracket & =0 & & \text { on } \gamma \in \Gamma_{\text {int }} .
\end{aligned}
$$

Consequently, the strong form associated with (14) is the following:

$$
\begin{aligned}
-\Delta e=r & \text { in } \cup_{k} \Omega_{k}, \\
\nabla e \cdot \boldsymbol{n}=l & \text { on } \Gamma_{\mathrm{N}}, \\
\llbracket \nabla e \cdot \boldsymbol{n}^{\gamma} \rrbracket=j & \text { on } \gamma \in \Gamma_{\mathrm{int}}, \\
e=0 & \text { on } \Gamma_{\mathrm{D}},
\end{aligned}
$$

where the strong interior residual is $r:=f+\Delta u_{H}$, the singular residual associated with flux discontinuities is represented by both the non-verification of the Neumann boundary conditions $l:=g-\nabla u_{H} \cdot n$ and the jump across the interelement boundaries $j:=-\llbracket \nabla u_{H} \cdot n \gamma \rrbracket$.

\subsection{Dual formulation and complementary energy minimization}

The dual formulation of the problem consists in introducing a new variable approximating the flux, $q \approx \nabla$ e. This approximated flux is said to be statically admissible (in analogy with the mechanical problem) if $q \in\left[\mathcal{L}_{2}(\Omega)\right]^{2}$ and fulfills equations (17a), (17b) and (17c), that is

$$
-\nabla \cdot q=r \quad \text { in } \cup_{k} \Omega_{k}
$$
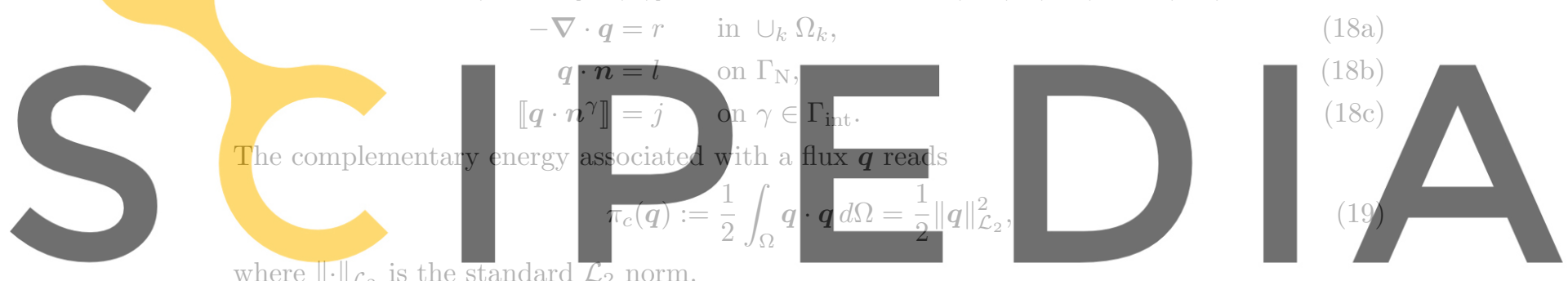

It is easily shown that any statically admissible flux (that is, fulfilling (18)) is such that

Register for free at https//www.scipediaçam to downlgad the version without the watermark

Moreover, if $q$ ranges in the proper functional space (of derivatives of functions in $\mathcal{V}$ ) the minimum of the complementary energy is precisely achieved by the error flux $\boldsymbol{q}=\boldsymbol{\nabla} e$.

A brief sketch of the proof of this essential property is recalled here.

A new bilinear form $a^{\mathrm{c}}(\cdot, \cdot)$ is introduced expressed in terms of flux quantities:

$$
a^{\mathrm{c}}(\boldsymbol{q}, \boldsymbol{p}):=\int_{\Omega} \boldsymbol{q} \cdot \boldsymbol{p} d \Omega .
$$

Note that $a^{\mathrm{c}}(\cdot, \cdot)$ is equivalent to $a(\cdot, \cdot)$ in the sense that

$$
a(u, v)=a^{\mathrm{c}}(\nabla u, \nabla v)
$$

and also that it is related with the complementary energy because

$$
\pi_{c}(\boldsymbol{q})=\frac{1}{2} a^{\mathrm{c}}(\boldsymbol{q}, \boldsymbol{q})
$$

A statically admissible approximation $\boldsymbol{q}$ of the error flux fulfills (18) and consequently also its weak counterpart (14) (replacing $\nabla e$ by $\boldsymbol{q}$ ). This reads

$$
a^{\mathrm{c}}(\boldsymbol{q}, \nabla v)=R(v) \quad \text { for all } v \in \mathcal{V} .
$$

Thus, for $v=e$, using (5) and (20) yields

$$
a^{\mathrm{c}}(\boldsymbol{q}, \nabla e)=R(e)=a(e, e)=\|e\|^{2} .
$$

Finally, using the Cauchy-Schwartz inequality, the upper bound property follows

$$
\|e\|^{2}=a^{\mathrm{c}}(\boldsymbol{q}, \nabla e) \leq\|\boldsymbol{q}\|_{\mathcal{L}_{2}}\|\nabla e\|_{\mathcal{L}_{2}}=\|\boldsymbol{q}\|_{\mathcal{L}_{2}}\|e\| \quad \text { that is } \quad\|e\| \leq\|\boldsymbol{q}\|_{\mathcal{L}_{2}},
$$

because $\|\cdot\|_{\mathcal{L}_{2}}$, in this case, coincides with the norm induced by $a^{\mathrm{c}}(\cdot, \cdot)$. 


\subsection{Domain decomposition of the dual formulation of the error equation}

As indicated in the previous section, the idea behind the flux-free approach is to split the force term (the r.h.s. of the equilibrium equation (5)) weighting it with the linear interpolation functions $\phi_{i}$, and restricting each problem to the support $\omega_{i}$. Here, this strategy is directly reproduced with the dual formulation summarized in equation (18). Actually, the same conclusion can also be derived by dualizing the strong form of the flux-free residual local problem (8).

The idea is then to split an approximation $\hat{\boldsymbol{q}}$ to $\boldsymbol{q}=\nabla e$ into local contributions $\hat{\boldsymbol{q}}^{i}$ associated with the stars, that is

$$
\hat{\boldsymbol{q}}=\sum_{i=1}^{n_{\mathrm{np}}} \hat{\boldsymbol{q}}^{i} .
$$

Each contribution $\hat{\boldsymbol{q}}^{i}$ is defined to be such that the following equations are fulfilled:

$$
\begin{aligned}
-\boldsymbol{\nabla} \cdot \hat{\boldsymbol{q}}^{i} & =\phi_{i} r & & \text { in } \omega_{i}, \\
\hat{q}^{i} \cdot n & =0 & & \text { on } \gamma \in \partial \omega_{i}-\left\{\Gamma_{\mathrm{N}} \cup \Gamma_{\mathrm{D}}\right\}, \\
\hat{q}^{i} \cdot n & =\phi_{i} l & & \text { on } \partial \omega_{i} \cap \Gamma_{\mathrm{N}}, \\
\llbracket \hat{q}^{i} \cdot n^{\gamma} \rrbracket & =\phi_{i j} & & \text { on } \gamma \in \Gamma_{\mathrm{int}} \cap \omega_{i},
\end{aligned}
$$

where here $n$ is the unit outward normal to $\partial \omega^{i}$. Note that this is nothing but the local version

of (18) with the r.h.s. weighted by $\phi_{i}$. The partition of unity property (6) ensures that if every

$\hat{q}^{i}$ fulfills (23), then $\hat{q}$ defined in (22) fulfills (18). Consequently, the norm of this solution
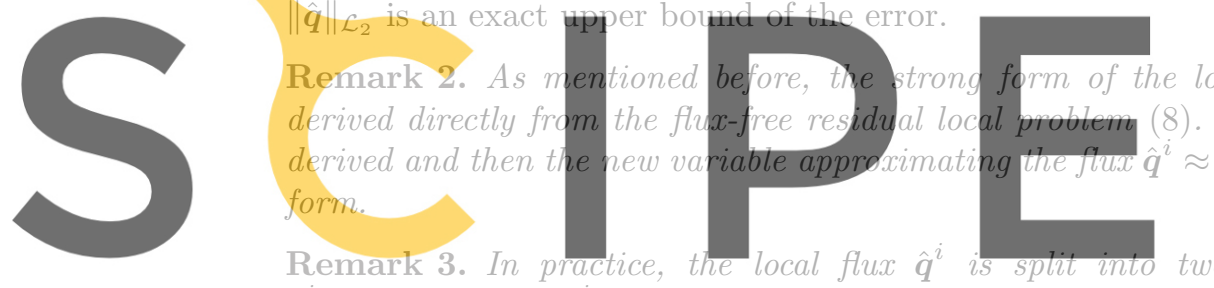

$\hat{\boldsymbol{q}}_{h}^{i}=\phi_{i} \nabla u_{H}$ and $\hat{q}_{*}^{i}$ is the solution of

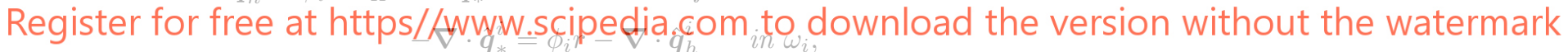

$$
\begin{aligned}
& \hat{q}_{*}^{i} \cdot n=\hat{q}_{h}^{i} \cdot n \\
& \hat{\boldsymbol{q}}_{*}^{i} \cdot \boldsymbol{n}=\phi_{i} l+\hat{\boldsymbol{q}}_{h}^{i} \cdot \boldsymbol{n} \quad \text { on } \partial \omega_{i} \cap \Gamma_{\mathrm{N}}, \\
& \llbracket \hat{\boldsymbol{q}}_{*}^{i} \cdot \boldsymbol{n}^{\gamma} \rrbracket=\phi_{i} j+\llbracket \hat{\boldsymbol{q}}_{h}^{i} \cdot \boldsymbol{n}^{\gamma} \rrbracket \quad \text { on } \gamma \in \Gamma_{\text {int }} \cap \omega_{i},
\end{aligned}
$$

which rearranging terms yields

$$
\begin{aligned}
-\boldsymbol{\nabla} \cdot \hat{\boldsymbol{q}}_{*}^{i} & =\phi_{i} f-\nabla \phi_{i} \cdot \nabla u_{H} & & \text { in } \omega_{i}, \\
\hat{\boldsymbol{q}}_{*}^{i} \cdot \boldsymbol{n} & =0 & & \text { on } \gamma \in \partial \omega_{i}-\left\{\Gamma_{\mathrm{N}} \cup \Gamma_{\mathrm{D}}\right\}, \\
\hat{\boldsymbol{q}}_{*}^{i} \cdot \boldsymbol{n} & =\phi_{i} g & & \text { on } \partial \omega_{i} \cap \Gamma_{\mathrm{N}}, \\
\llbracket \hat{\boldsymbol{q}}_{*}^{i} \cdot \boldsymbol{n}^{\gamma} \rrbracket & =0 & & \text { on } \gamma \in \Gamma_{\mathrm{int}} \cap \omega_{i} .
\end{aligned}
$$

Note that computing $\hat{\boldsymbol{q}}_{*}^{i}$ does not require computing the flux of $u_{H}$ at interelementary edges. Moreover, equations (24c) enforce that $\hat{\boldsymbol{q}}_{*}^{i}$ is continuous in the normal direction at the edges inside the star $\omega_{i}$, which implementation is simpler to implement than enforcing a prescribed jump on the normal direction (23c).

Under the general assumption that fields $f$ and $g$ are piecewise polynomial, it is possible to determine - amongst all the dual estimates $\hat{\boldsymbol{q}}_{*}^{i}$ fulfilling equations (24) - a piecewise polynomial solution of (24). That is, for a given suitable interpolation degree $q$, it is possible to find $\hat{\boldsymbol{q}}_{*}^{i} \in\left[\widehat{\mathbb{P}}^{q}\left(\omega_{i}\right)\right]^{2}$ verifying equation (24) where

$$
\widehat{\mathbb{P}}^{q}\left(\omega_{i}\right):=\left\{v \in \mathcal{L}^{2}\left(\omega_{i}\right) \text { s.t. for every } \Omega_{k} \subset \omega_{i},\left.v\right|_{\Omega_{k}} \in \mathbb{P}^{q}\left(\Omega_{k}\right)\right\} .
$$


In this case, the global estimate is a piecewise polynomial field, namely $\hat{\boldsymbol{q}} \in\left[\widehat{\mathbb{P}}^{q}(\Omega)\right]^{2}$.

Remark 4. A locally statically admissible flux $\hat{\boldsymbol{q}}^{i}$ fulfills (23) and consequently also its weak counterpart (8) (replacing $\nabla e_{\omega_{i}}$ by $\hat{\boldsymbol{q}}^{i}$ ). This reads

$$
a_{\omega_{i}}^{\mathrm{c}}\left(\hat{\boldsymbol{q}}^{i}, \nabla v\right)=R\left(\phi_{i} v\right)=a_{\omega_{i}}\left(e_{\omega_{i}}, v\right) \quad \text { for all } v \in \mathcal{H}^{1}\left(\omega_{i}\right)
$$

Moreover, if $\hat{\boldsymbol{q}}^{i}$ ranges in the proper functional space, the minimum of the local complementary energy

$$
\int_{\omega_{i}} \hat{\boldsymbol{q}}^{i} \cdot \hat{\boldsymbol{q}}^{i} d \Omega=a_{\omega_{i}}^{\mathrm{c}}\left(\hat{\boldsymbol{q}}^{i}, \hat{\boldsymbol{q}}^{i}\right)
$$

is achieved precisely by $\hat{\boldsymbol{q}}^{i}=\nabla e_{\omega_{i}}$.

\section{NUMERICAL IMPLEMENTATION}

\subsection{Piecewise polynomial representation for $\hat{\boldsymbol{q}}_{*}^{i}$}

The unknown $\hat{q}_{*}^{i}$ of problem (24) is taken to be polynomial of degree $q$ in each element of $\omega_{i}$, thus, $\hat{\boldsymbol{q}}_{*}^{i}$ may present discontinuities at the internal edges of the star $\omega_{i}$. Let $K_{i}=$ $\left\{k_{1}, k_{2}, \ldots, k_{m}\right\}$ be the set of indices of the elements lying in $\omega_{i}$, that is, such that

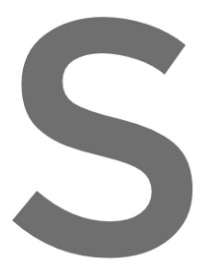

$$
\bar{\omega}_{i}=\cup_{k \in K_{i}} \bar{\Omega}_{k},
$$

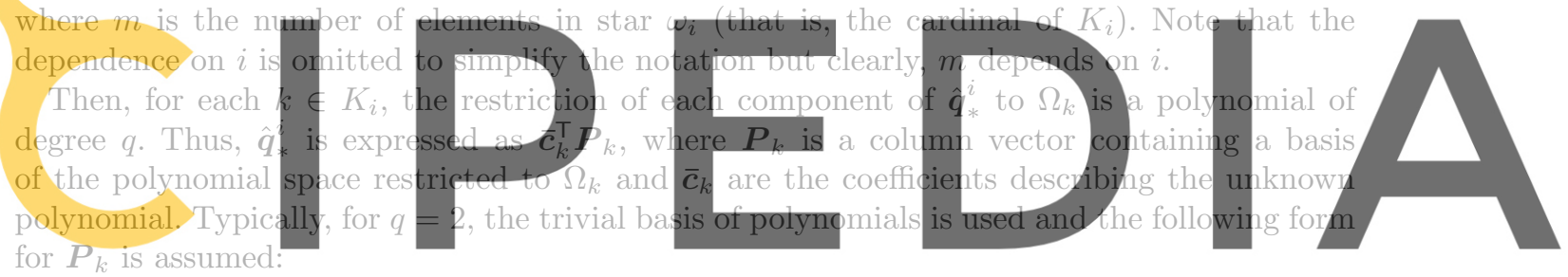

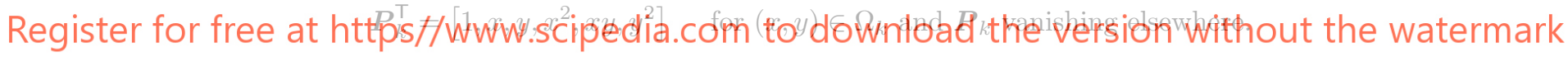

Note that, in practice, all vectors $\boldsymbol{P}_{k}$ are identical, except for where they are supported.

Then, the representation for $\hat{\boldsymbol{q}}_{*}^{i}$ is

$$
\hat{\boldsymbol{q}}_{*}^{i}=\sum_{k \in K_{i}} \overline{\boldsymbol{c}}_{k}^{\top} \boldsymbol{P}_{k}
$$

In order to account for the vectorial character of $\hat{\boldsymbol{q}}_{*}^{i}$ (in $2 \mathrm{D}$ a vector of two components), the coefficients $\overline{\boldsymbol{c}}_{k}$ must be arranged in a two-column matrix with the following shape

$$
\overline{\boldsymbol{c}}_{k}^{\top}=\left[\begin{array}{c}
\left(\boldsymbol{c}_{k}^{x}\right)^{\top} \\
\left(\boldsymbol{c}_{k}^{y}\right)^{\top}
\end{array}\right]=\left[\begin{array}{cccc}
c_{k}^{x, 1} & c_{k}^{x, 2} & \ldots & c_{k}^{x, N} \\
c_{k}^{y, 1} & c_{k}^{y, 2} & \ldots & c_{k}^{y, N}
\end{array}\right]
$$

$N$ being the number of components of $\boldsymbol{P}_{k}$, that is, in $2 \mathrm{D} N=(q+1)(q+2) / 2$. Thus, the total number of unknowns in every star is $2 m N$.

In practice, the following equivalent representation is used

$$
\hat{\boldsymbol{q}}_{*}^{i}=\sum_{k \in K_{i}} \boldsymbol{Q}_{k} \boldsymbol{c}_{k}
$$

where

$$
\boldsymbol{c}_{k}^{\top}=\left[\begin{array}{lll}
\left(\boldsymbol{c}_{k}^{x}\right)^{\top} & \left(\boldsymbol{c}_{k}^{y}\right)^{\top}
\end{array}\right]=\left[\begin{array}{lllll}
c_{k}^{x, 1} c_{k}^{x, 2} \ldots & c_{k}^{x, N} & c_{k}^{y, 1} c_{k}^{y, 2} \ldots & c_{k}^{y, N}
\end{array}\right] \quad \text { and } \quad \boldsymbol{Q}_{k}=\left[\begin{array}{cc}
\boldsymbol{P}_{k}^{\top} & \mathbf{0} \\
\mathbf{0} & \boldsymbol{P}_{k}^{\top}
\end{array}\right] .
$$


All these unknowns are collected in a column vector $\boldsymbol{c}$

$$
\begin{aligned}
& \boldsymbol{c}^{\top}=\left[\boldsymbol{c}_{k_{1}}^{\top} \boldsymbol{c}_{k_{2}}^{\top} \ldots \boldsymbol{c}_{k_{m}}^{\top}\right]=\left[\left(\boldsymbol{c}_{k_{1}}^{x}\right)^{\top}\left(\boldsymbol{c}_{k_{1}}^{y}\right)^{\top}\left(\boldsymbol{c}_{k_{2}}^{x}\right)^{\top}\left(\boldsymbol{c}_{k_{2}}^{y}\right)^{\top} \ldots\left(\boldsymbol{c}_{k_{m}}^{x}\right)^{\top}\left(\boldsymbol{c}_{k_{m}}^{y}\right)^{\top}\right] \\
& =\left[\begin{array}{lllllllllll}
c_{k_{1}}^{x, 1} c_{k_{1}}^{x, 2} \ldots & c_{k_{1}}^{x, N} & c_{k_{1}}^{y, 1} c_{k_{1}}^{y, 2} \ldots & c_{k_{1}}^{y, N} & \ldots & c_{k_{m}}^{x, 1} c_{k_{m}}^{x, 2} \ldots & c_{k_{m}}^{x, N} & c_{k_{m}}^{y, 1} c_{k_{m}}^{y, 2} \ldots & c_{k_{m}}^{y, N}
\end{array}\right] .
\end{aligned}
$$

Thus, the following compact form for the polynomial representation (25) is introduced

$$
\hat{\boldsymbol{q}}_{*}^{i}=\boldsymbol{Q c}
$$

where

$$
\boldsymbol{Q}=\left[\boldsymbol{Q}_{k_{1}} \boldsymbol{Q}_{k_{2}} \ldots \boldsymbol{Q}_{k_{m}}\right]=\left[\begin{array}{ccccccc}
\boldsymbol{P}_{k_{1}}^{\top} & \mathbf{0} & \boldsymbol{P}_{k_{2}}^{\top} & \mathbf{0} & \ldots & \boldsymbol{P}_{k_{m}}^{\top} & \mathbf{0} \\
\mathbf{0} & \boldsymbol{P}_{k_{1}}^{\top} & \mathbf{0} & \boldsymbol{P}_{k_{2}}^{\top} & \ldots & \mathbf{0} & \boldsymbol{P}_{k_{m}}^{\top}
\end{array}\right]
$$

Note that the dependence on $i$ of the vector $\boldsymbol{c}$ and matrix $\boldsymbol{Q}$ is omitted to simplify the notation but clearly, $\boldsymbol{c}$ and $\boldsymbol{Q}$ depend on $i$.

\subsection{Imposing the divergence condition to the polynomial flux \\ In every star $\omega_{i}$, the restrictions (24) have to be imposed to the trial fluxes defined in (25) or} (27).

Equation (24a) sets the pointwise value of the flux divergence, $\nabla \cdot \hat{\boldsymbol{q}}_{*}^{i}$. Following (27) the flux divergence is

$$
\nabla \cdot \hat{q}_{*}^{i}=(\nabla \cdot Q) c=\left[\partial_{x} P_{k_{1}}^{\top} \partial_{y} P_{k_{1}}^{\top} \partial_{x} \boldsymbol{P}_{k_{2}}^{\top} \partial_{y} \boldsymbol{P}_{k_{2}}^{\top} \cdots \partial_{x} \boldsymbol{P}_{k_{m}}^{\top} \partial_{y} \boldsymbol{P}_{k_{m}}^{\top}\right] c .
$$
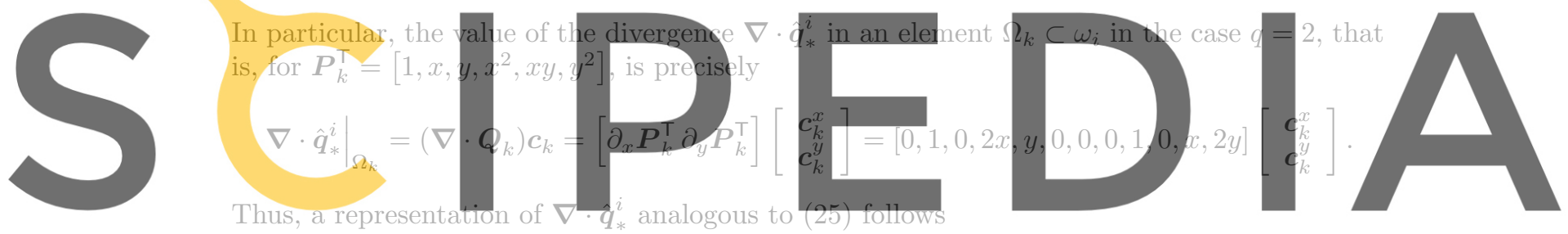

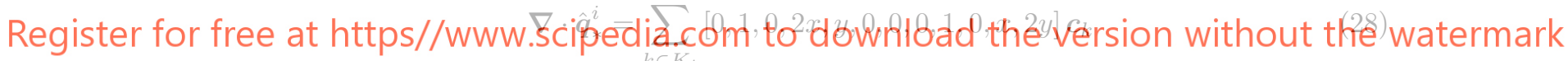

\section{that is straightforwardly generalized to higher polynomial degrees.}

The r.h.s. term of $(24 \mathrm{a}) \phi_{i} f-\nabla \phi_{i} \cdot \nabla u_{H}$, is assumed to be polynomial in every element of the mesh. This assumption is not restrictive because it holds if the source term is polynomial in each element of the mesh (not necessarily the same polynomial in the whole domain). Thus, it suffices to take $q$ large enough such that equation (24a) can be fulfilled by the trial flux $\hat{\boldsymbol{q}}_{*}^{i}$. For instance, for a piecewise constant source term $s$, the r.h.s. term of (24a) is linear and $q=2$ suffices.

As shown in (28), for $q=2$ the divergence of the flux is one degree less than the flux itself. In particular, in this case it is linear and therefore imposing this function to be equal to some other linear function results in 3 equations. Indeed, if the r.h.s. of (24a) in the element $\Omega_{k}$ is a linear function $f_{k}:=\phi_{i} f-\left.\nabla \phi_{i} \cdot \nabla u_{H}\right|_{\Omega_{k}}=a_{k}^{c}+a_{k}^{x} x+a_{k}^{y} y$, the condition $\left.\nabla \cdot \hat{\boldsymbol{q}}_{*}^{i}\right|_{\Omega_{k}}=f_{k}$ turns out to be

$$
\boldsymbol{c}_{k}^{x, 2}+2 x \boldsymbol{c}_{k}^{x, 4}+y \boldsymbol{c}_{k}^{x, 5}+\boldsymbol{c}_{k}^{y, 3}+x \boldsymbol{c}_{k}^{y, 5}+2 y c_{k}^{y, 6}=a_{k}^{c}+a_{k}^{x} x+a_{k}^{y} y,
$$

which in matrix form is

$$
\left[\begin{array}{llllllllllll}
0 & 1 & 0 & 0 & 0 & 0 & 0 & 0 & 1 & 0 & 0 & 0 \\
0 & 0 & 0 & 2 & 0 & 0 & 0 & 0 & 0 & 0 & 1 & 0 \\
0 & 0 & 0 & 0 & 1 & 0 & 0 & 0 & 0 & 0 & 0 & 2
\end{array}\right] \boldsymbol{c}_{k}=\left[\begin{array}{c}
a_{k}^{c} \\
a_{k}^{x} \\
a_{k}^{y}
\end{array}\right]
$$

Note that the previous equations are equivalent to imposing the equality $\left(\boldsymbol{\nabla} \cdot \boldsymbol{Q}_{k}\right) \boldsymbol{c}_{k}=f_{k}$ at the three points $x=0$ and $y=0, x=1$ and $y=0$ and $x=0$ and $y=1$. A different option to 
obtain the three required equations is to impose $\left(\nabla \cdot Q_{k}\right) c_{k}=f_{k}$ at the three vertex nodes of the triangle $\Omega_{k}$ (or at three linearly independent points (not aligned) inside the triangle, for instance, at three linearly independent gauss points).

In general the number of linear restrictions resulting from (24a) is $(q+1) q / 2$ in each element of the star $\omega_{i}$. Thus, recalling that the number of elements in the star is denoted by $m$, the $m(q+1) q / 2$ linear restrictions associated with (24a) are written as

$$
D_{1} c=d_{1},
$$

where $D_{1}$ is a $m(q+1) q / 2 \times 2 m N$ matrix and $\boldsymbol{d}_{1}$ is a column vector of $m(q+1) q / 2$ components.

\subsection{Equilibrium restrictions on the boundary of the star}

Equations (24b) and (24c) prescribe the normal flux on the boundary of the star that does not intersect the Dirichlet boundary $\Gamma_{\mathrm{D}}$. The only difference between (24b) and (24c) is that the prescribed value may not be zero along the Neumann boundary $\Gamma_{\mathrm{N}}$. Note that the value of the normal fux at the Dirichlet boundaries is not prescribed.

For a typical star corresponding to an interior node, the number of edges on the boundary of $\omega_{i}$ coincides with the number of elements in the star, $m$. If the center of the star is a boundary node, the number of edges on the boundary of $\omega_{i}$ is $m+2$. In the following, the number of edges on the boundary that do not lie on the Dirichlet boundary $\Gamma_{\mathrm{D}}$ is denoted by $m_{b}$.

The expression of the trial flux on an edge of element $\Omega_{k}$ reads

$$
\left.\hat{q}_{*}^{i} \cdot n\right|_{\Omega_{k}}=\left[n_{x} c_{k}^{x}+n_{y} c_{k}^{y}\right]^{\top} P_{k}=\left[n_{x} P_{k}^{\top}+n_{y} P_{k}^{\top}\right] c_{k}
$$
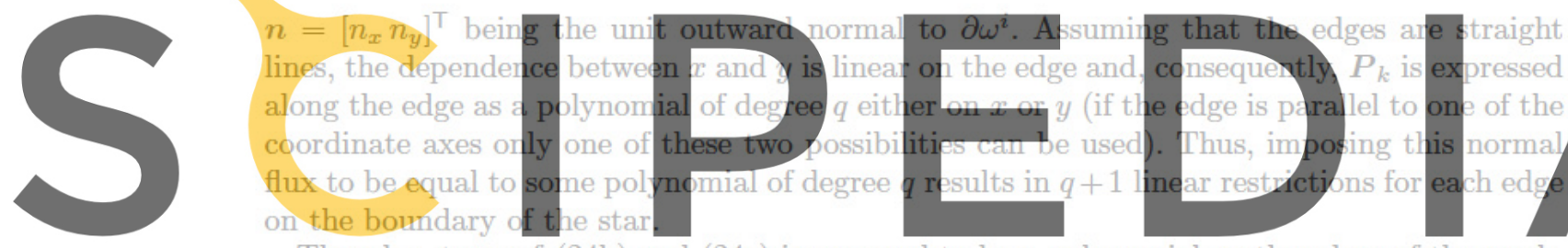

The r.h.s. term of (24b) and (24c) is assumed to be a polynomial on the edges of the mesh.

suffices to take $q$ large enough such that equations (24b) and (24c) can be fulfilled by the trial

flux $\hat{\boldsymbol{q}}_{*}^{i}$. For instance, for a piecewise constant $g$, the r.h.s. of $(24 c)$ is linear and $q=1$ suffices.

Therefore, if the source term $f$ and the Neumann boundary conditions $g$ are assumed to be piecewise constant, $q=2$ suffices to impose equations (24a), (24b) and (24c). In particular, in this case imposing the normal flux $\hat{\boldsymbol{q}}_{*}^{i} \cdot \boldsymbol{n}$ to be equal to some polynomial of degree $q=2$ results in 3 linear restrictions for each edge on the boundary of the star. Indeed, given an edge

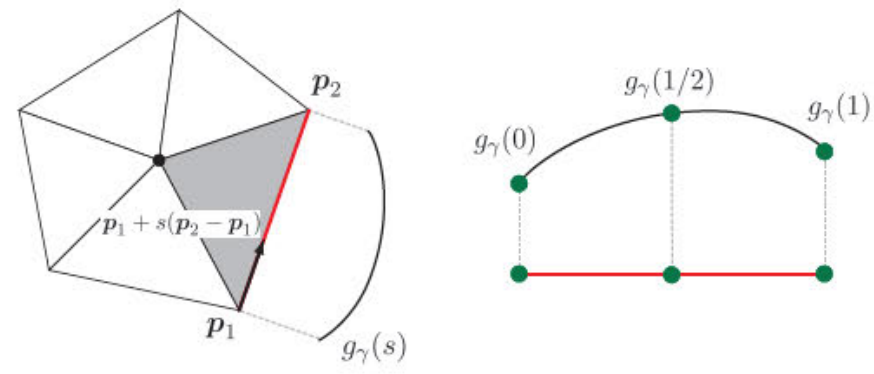

Figure 1. Equilibrium restrictions on the boundary of the star for $q=2$.

$\gamma \in \partial \omega_{i}$ and denoting by $\boldsymbol{p}_{1}$ and $\boldsymbol{p}_{2}$ the starting and ending points of this edge, the points on $\gamma$ may be described using a parameter $s \in[0,1]$ as $p(s)=p_{1}+s\left(p_{2}-p_{1}\right)$. Thus, imposing $\hat{\boldsymbol{q}}_{*}^{i} \cdot \boldsymbol{n}=g(s)$ yields

$$
\left[n_{x} \boldsymbol{P}_{k}(\boldsymbol{p}(s))^{\mathrm{\top}}+n_{y} \boldsymbol{P}_{k}(\boldsymbol{p}(s))^{\mathrm{\top}}\right] \boldsymbol{c}_{k}=g(s) .
$$


Since both the r.h.s. and the l.h.s. are polynomials of degree 2 on $s$, the three equations imposing the fluxes at the edge $\gamma$ may be derived taking $s=0,1 / 2$ and 1 in the previous equation, see figure 1 . Note that any other three points could be chosen to impose the flux condition.

Thus, the corresponding equations on the unknown coefficients $c$ corresponding to (24b) and $(24 \mathrm{c})$ read

$$
D_{2} c=d_{2},
$$

where $D_{2}$ is a $m_{b}(q+1) \times 2 m N$ matrix and $d_{2}$ is a column vector of $m_{b}(q+1)$ components.

\subsection{Equilibrium restrictions on the interior edges of the star}

Equation (24d) enforces the normal continuity of the flux jump across the interior edges of the star. The number of interior edges in the star is denoted by $m_{i}$. In the typical case of a star associated with an interior node, $m_{i}$ coincides with $m$ (and $m_{b}$ ), otherwise $m_{i}=m-1$.

The flux jump across the edge $\gamma$ belonging to elements $\Omega_{k_{1}}$ and $\Omega_{k_{2}}$, that is $\gamma=\partial \Omega_{k_{1}} \cap \partial \Omega_{k_{2}}$, reads

$$
\begin{aligned}
\llbracket \hat{\boldsymbol{q}}_{*}^{i} \cdot \boldsymbol{n}^{\gamma} \rrbracket & =\left.\left(\boldsymbol{n}^{\gamma} \cdot \boldsymbol{n}_{k_{1}}^{\gamma}\right) \hat{\boldsymbol{q}}_{*}^{i}\right|_{\Omega_{k_{1}}} \cdot \boldsymbol{n}^{\gamma}+\left.\left(\boldsymbol{n}^{\gamma} \cdot \boldsymbol{n}_{k_{2}}^{\gamma}\right) \hat{\boldsymbol{q}}_{*}^{i}\right|_{\Omega_{k_{2}}} \cdot \boldsymbol{n}^{\gamma} \\
& =\left[n_{x} \boldsymbol{P}_{k_{1}}^{\top}+n_{y} \boldsymbol{P}_{k_{1}}^{\top}\right] \boldsymbol{c}_{k_{1}}-\left[n_{x} \boldsymbol{P}_{k_{2}}^{\top}+n_{y} \boldsymbol{P}_{k_{2}}^{\top}\right] \boldsymbol{c}_{k_{2}}
\end{aligned}
$$

where $n^{\gamma}=\left[n_{x} n_{y}\right]^{\top}$ is the unit normal associated with the edge $\gamma$ which has been assumed to coincide with $\boldsymbol{n}_{k_{1}}^{\gamma}$, and thus $\boldsymbol{n}^{\gamma} \cdot \boldsymbol{n}_{k_{1}}^{\gamma}=1$ and $\boldsymbol{n}^{\gamma} \cdot \boldsymbol{n}_{k_{2}}^{\gamma}=-1$, see figure 2 .

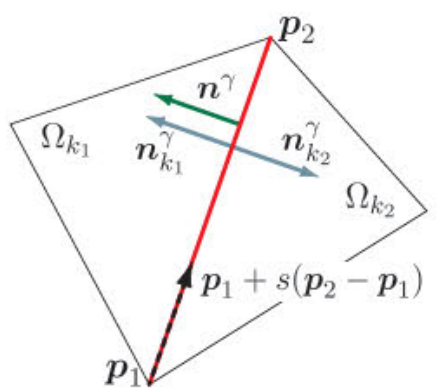

Figure 2. Equilibrium restrictions on the interior edges of the star.

As stated in the previous section, the restriction of the flux to a straight edge is a univariate polynomial of degree $q$. Thus the previous equation which enforces the normal continuity of the flux jump across the edge $\gamma$ results in $q+1$ equations relating $c_{k_{1}}$ and $c_{k_{2}}$.

Indeed, as in the previous section, the points on an edge $\gamma$ may be described using a parameter $s \in[0,1]$ as $\boldsymbol{p}(s)=\boldsymbol{p}_{1}+s\left(\boldsymbol{p}_{2}-\boldsymbol{p}_{1}\right)$ where $\boldsymbol{p}_{1}$ and $\boldsymbol{p}_{2}$ are the starting and ending points of $\gamma$. Thus, imposing $\llbracket \hat{q}_{*}^{i} \cdot n^{\gamma} \rrbracket=0$ yields

$$
\left[n_{x} \boldsymbol{P}_{k_{1}}(p(s))^{\top}+n_{y} \boldsymbol{P}_{k_{1}}(p(s))^{\top}\right] c_{k_{1}}-\left[n_{x} \boldsymbol{P}_{k_{2}}(p(s))^{\top}+n_{y} \boldsymbol{P}_{k_{2}}(p(s))^{\top}\right] c_{k_{2}}=0 .
$$

Since both the r.h.s. and the l.h.s. are polynomials of degree $q$ on $s$, the $q+1$ equations imposing the fluxes at the edge $\gamma$ may be derived taking $q+1$ different values for $s$ in the previous equation. In particular, for $q=2, s=0,1 / 2$ and 1 may be used.

This results in a set of linear restrictions for $c$ :

$$
D_{3} c=d_{3}
$$

where $D_{3}$ is a $m_{i}(q+1) \times 2 m N$ matrix and $d_{3}=0$ is a null column vector of $m_{i}(q+1)$ components. 


\subsection{Imposing statical admissibility to $\hat{\boldsymbol{q}}_{*}^{i}$}

Putting together the three sets of linear equations corresponding to the interior equilibrium and the edge equilibrium for both the boundary edges and the interior edges results in a compact form

$$
D c=d,
$$

where $\boldsymbol{D}$ is obtained appending $\boldsymbol{D}_{1}, \boldsymbol{D}_{2}$ and $\boldsymbol{D}_{3}$ and it is a rectangular matrix with $2 m N$ columns and $n_{\text {rest }}$ rows, with

$$
n_{\text {rest }}:=m(q+1) q / 2+\left(m_{b}+m_{i}\right)(q+1) .
$$

Note that $n_{\text {rest }}$ stands for the number of restrictions.

Remark 5. In the case where the star $\omega_{i}$ does not intersect the Dirichlet boundary $\Gamma_{\mathrm{D}}$ the previous equations are not linearly independent, since one of the equations is redundant. The number of linearly independent equations is in this case $n_{\text {rest }}-1$. This is easily shown by considering that

$$
\begin{aligned}
\int_{\omega_{i}} \hat{\boldsymbol{q}}_{*}^{i} \cdot \nabla v d \Omega & =\int_{\omega_{i}}\left[\nabla \cdot\left(\hat{\boldsymbol{q}}_{*}^{i} v\right)-\boldsymbol{\nabla} \cdot \hat{\boldsymbol{q}}_{*}^{i} v\right] d \Omega=-\int_{\omega_{i}} \boldsymbol{\nabla} \cdot \hat{\boldsymbol{q}}_{*}^{i} v d \Omega+\sum_{k \in K_{i}} \int_{\Omega_{k}} \boldsymbol{\nabla} \cdot\left(\hat{\boldsymbol{q}}_{*}^{i} v\right) d \Omega \\
& =-\int_{\omega_{i}} \boldsymbol{\nabla} \cdot \hat{\boldsymbol{q}}_{*}^{i} v d \Omega+\sum_{k \in K_{i}} \sum_{\gamma \in \partial \Omega_{k}} \int_{\gamma} \hat{\boldsymbol{q}}_{*}^{i} \cdot \boldsymbol{n}_{k}^{\gamma} v d \Gamma \\
& =-\int_{\omega_{i}} \boldsymbol{\nabla} \cdot \hat{\boldsymbol{q}}_{*}^{i} v d \Omega+\sum_{\gamma \in \partial \omega_{i}} \int_{\gamma} \hat{\boldsymbol{q}}_{*}^{i} \cdot \boldsymbol{n} v d \Gamma+\sum_{\gamma \in \Gamma_{\mathrm{int}} \cap \omega_{i}} \int_{\gamma} \llbracket \hat{\boldsymbol{q}}_{*}^{i} \cdot \boldsymbol{n}^{\gamma} \rrbracket v d \Gamma
\end{aligned}
$$

from which taking $v=1$ yields

$$
-\int_{\omega_{i}} \boldsymbol{\nabla} \cdot \hat{\boldsymbol{q}}_{*}^{i} d \Omega+\sum_{\gamma \in \partial \omega_{i}} \int_{\gamma} \hat{\boldsymbol{q}}_{*}^{i} \cdot \boldsymbol{n} d \Gamma+\sum_{\gamma \in \Gamma_{\mathrm{int}} \cap \omega_{i}} \int_{\gamma} \llbracket \hat{\boldsymbol{q}}_{*}^{i} \cdot \boldsymbol{n}^{\gamma} \rrbracket d \Gamma=0 .
$$

Thus, equations in (29) that are setting the values of $\boldsymbol{\nabla} \cdot \hat{\boldsymbol{q}}_{*}^{i}$ in $\omega_{i}, \hat{\boldsymbol{q}}_{*}^{i} \cdot \boldsymbol{n}_{k}^{\gamma}$ on $\partial \omega_{i}$ and $\llbracket \hat{\boldsymbol{q}}_{*}^{i} \cdot \boldsymbol{n}^{\gamma} \rrbracket$ on $\Gamma_{\text {int }} \cap \omega_{i}$ are not linearly independent because they are linked by the previous equation. One of the equations in (29) is recovered as a linear combination of the others and therefore the rank of $\boldsymbol{D}$ is $n_{\text {rest }}-1$.

The undetermined system of equations (29) is compatible, that is it admits solutions. This is proven using the same rationale as that followed in to analyze the solvability of equation (8) which is based on the fact that $R\left(\phi_{i}\right)=0$ (for nodes $i$ not on the Dirichlet boundary $\Gamma_{\mathrm{D}}$ ). Indeed, using equations (24) and (11) with $v=1$

$$
\begin{aligned}
0 & =-\int_{\omega_{i}} \boldsymbol{\nabla} \cdot \hat{\boldsymbol{q}}_{*}^{i} d \Omega+\sum_{\gamma \in \partial \omega_{i}} \int_{\gamma} \hat{\boldsymbol{q}}_{*}^{i} \cdot \boldsymbol{n} d \Gamma+\sum_{\gamma \in \Gamma_{\mathrm{int}} \cap \omega_{i}} \int_{\gamma} \llbracket \hat{\boldsymbol{q}}_{*}^{i} \cdot \boldsymbol{n}^{\gamma} \rrbracket d \Gamma \\
& =\int_{\omega_{i}}\left[\phi_{i} f-\nabla \phi_{i} \cdot \nabla u_{H}\right] d \Omega+\sum_{\gamma \in \partial \omega_{i} \cap \Gamma_{\mathrm{N}}} \int_{\gamma} \phi_{i} g d \Gamma=R\left(\phi_{i}\right)
\end{aligned}
$$

which holds for any shape function $\phi_{i}$ not intersecting the Dirichlet boundary $\Gamma_{\mathrm{D}}$.

The conditions $(24)$ to obtain a statically admissible field $\hat{\boldsymbol{q}}_{*}^{i}$ which is piecewise polynomial of degree $q$ inside each element of the star yield equations (29). The total number of degrees of freedom are $2 m N=m(q+1)(q+2)$ and equations (29) determine $n_{\text {rest }}-1$ linearly independent conditions in the case where the star does not intersect the Dirichlet boundary, $\partial \omega_{i} \cap \Gamma_{\mathrm{D}}=\emptyset$, or $n_{\text {rest }}$ otherwise.

For instance, in the case $q=2$ the total number of degrees of freedom is $12 \mathrm{~m}$. The number of conditions given by equation (29) depends on the following cases, see figure 3 :

(i) star corresponding to an interior node,

(ii) the center of the star is a boundary node and its boundary intersects only the Neumann boundary, 

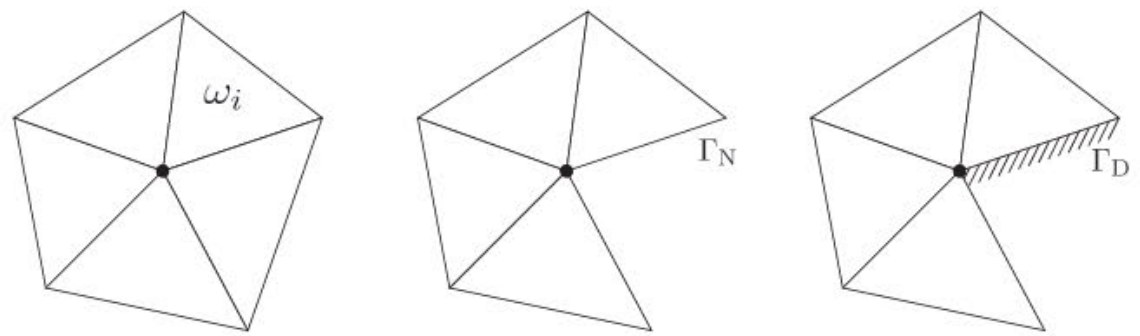

Figure 3. Star $\omega_{i}$ : interior star (left), star intersecting only the Neumann boundary $\Gamma_{\mathrm{N}}$ (center), star intersecting the Dirichlet boundary $\Gamma_{\mathrm{D}}$ (right).

(iii) the center of the star is a boundary node and its boundary intersects the Dirichlet boundary.

In the first case, equation (29) poses $3 m+6 m-1=9 m-1$ conditions since in this case $m=m_{b}=m_{i}$, and therefore, there are $3 m+1$ degrees of freedom which are not determined from the previous equations. In the second case, equation (29) poses $3 m+3(m+2+m-1)-1=$ $9 m+2$ conditions since in this case $m_{b}=m+2$ and $m_{i}=m-1$, and therefore, there are $3 m-2$ free degrees of freedom. Finally, in the third case, equation (29) poses $3 m+3\left(m+m_{b \mathrm{~N}}+m-1\right)=9 m+3\left(m_{b \mathrm{~N}}-1\right)$ conditions since in this case $m_{i}=m-1$ and $m_{b}=m+m_{b \mathrm{~N}}$ for $m_{b \mathrm{~N}}$ being the number of edges intersecting the Neumann boundary $\left(m_{b \mathrm{~N}}=0\right.$ or 1$)$. Therefore, there are $3 m+3\left(1-m_{b \mathrm{~N}}\right)$ free degrees of freedom.

It is worth noting that equations (29) in general do not uniquely determine the dual estimate $\hat{\boldsymbol{q}}_{*}^{i}$. These additional degrees of freedom are used to minimize the final bounds. This is discussed in the following section.

\subsection{Determination of the additional degrees of freedom - complementary energy minimization}

Any set $\hat{\boldsymbol{q}}_{*}^{i} i=1, \ldots, n_{\mathrm{np}}$ fulfilling equations (29) produces a global estimate

$$
\hat{\boldsymbol{q}}=\sum_{i=1}^{n_{\text {np }}} \hat{\boldsymbol{q}}^{i}=\sum_{i=1}^{n_{\text {np }}} \hat{\boldsymbol{q}}_{*}^{i}-\hat{\boldsymbol{q}}_{h}^{i},
$$

providing an upper bound for the energy norm of $e$,

$$
\|e\|^{2} \leq\|q\|_{\mathcal{L}_{2}}^{2}=\int_{\Omega} \hat{\boldsymbol{q}} \cdot \hat{\boldsymbol{q}} d \Omega=\int_{\Omega}\left(\sum_{i=1}^{n_{\mathrm{np}}} \hat{\boldsymbol{q}}^{i}\right) \cdot\left(\sum_{i=1}^{n_{\mathrm{np}}} \hat{\boldsymbol{q}}^{i}\right) d \Omega
$$

The determination of the estimates $\hat{\boldsymbol{q}}^{i}$ (or equivalently $\hat{\boldsymbol{q}}_{*}^{i}$ ) minimizing the upper bound for $\|e\|$ requires coupling all the local problems.

In this work two different approaches have been studied. The simplest and most straightforward criterion to select one of the multiple choices $\hat{q}^{i}$ is considered first: the local estimates $\hat{q}^{i}$ are required to minimize the local complementary energy

$$
\int_{\omega_{i}} \hat{\boldsymbol{q}}^{i} \cdot \hat{\boldsymbol{q}}^{i} d \Omega
$$

This is equivalent to selecting $\hat{q}^{i}$ which yields the best approximation of $\nabla e_{\omega_{i}}$ in the space $\left[\widehat{\mathbb{P}}^{q}\left(\omega_{i}\right)\right]^{2}$, see remark 4. Indeed,

$$
\left\|e_{\omega_{i}}\right\|_{\omega_{i}}^{2}=\int_{\omega_{i}} \nabla e_{\omega_{i}} \cdot \nabla e_{\omega_{i}} d \Omega=\min _{\substack{\hat{q}^{i} \in\left[\mathcal{L}^{2}\left(\omega_{i}\right)\right]^{2} \\ \hat{q}^{i} \text { fulfills }(23)}} \int_{\omega_{i}} \hat{q}^{i} \cdot \hat{q}^{i} d \Omega \leq \min _{\substack{\hat{q}^{i} \in\left[\widehat{\mathbb{P}}^{q}\left(\omega_{i}\right)\right]^{2} \\ \hat{q}^{i} \text { fulfills }(23)}} \int_{\omega_{i}} \hat{q}^{i} \cdot \hat{q}^{i} d \Omega .
$$

The second approach takes advantage of the fact that the final bound is

$$
\int_{\Omega} \hat{\boldsymbol{q}} \cdot \hat{\boldsymbol{q}} d \Omega=\int_{\Omega}\left(\sum_{i=1}^{n_{\mathrm{np}}} \hat{\boldsymbol{q}}^{i}\right) \cdot\left(\sum_{i=1}^{n_{\mathrm{np}}} \hat{\boldsymbol{q}}^{i}\right) d \Omega
$$


to minimizing the energy of the accumulated flux approximation.

The local estimates $\hat{\boldsymbol{q}}^{i}$ are computed using an arbitrary order of the elements, from $i=1$ to $i=n_{\mathrm{np}}$. Thus, given a star $\omega_{i}$, the estimates $\hat{\boldsymbol{q}}^{j}, j=1, \ldots, i-1$ are already computed, and the local estimate $\hat{\boldsymbol{q}}^{i}$ is chosen to minimize

$$
\int_{\Omega}\left(\sum_{j=1}^{i} \hat{\boldsymbol{q}}^{j}\right) \cdot\left(\sum_{j=1}^{i} \hat{\boldsymbol{q}}^{j}\right) d \Omega
$$

which is equivalent to minimize

$$
\int_{\omega_{i}}\left(\sum_{j=1}^{i} \hat{\boldsymbol{q}}^{j}\right) \cdot\left(\sum_{j=1}^{i} \hat{\boldsymbol{q}}^{j}\right) d \Omega
$$

because the degrees of freedom of $\hat{\boldsymbol{q}}^{i}$ only affect the contribution of the star $\omega_{i}$ to the total complementary energy.

5.6.1. Minimization of the local complementary energy The local complementary energy associated with $\hat{\boldsymbol{q}}^{i}$ is

$\int_{\omega_{i}} \hat{\boldsymbol{q}}^{i} \cdot \hat{\boldsymbol{q}}^{i} d \Omega=\int_{\omega_{i}}\left(\hat{\boldsymbol{q}}_{*}^{i}-\hat{\boldsymbol{q}}_{h}^{i}\right) \cdot\left(\hat{\boldsymbol{q}}_{*}^{i}-\hat{\boldsymbol{q}}_{h}^{i}\right) d \Omega=\int_{\omega_{i}} \hat{\boldsymbol{q}}_{*}^{i} \cdot \hat{\boldsymbol{q}}_{*}^{i} d \Omega+\int_{\omega_{i}} \hat{\boldsymbol{q}}_{h}^{i} \cdot \hat{\boldsymbol{q}}_{h}^{i} d \Omega-2 \int_{\omega_{i}} \hat{\boldsymbol{q}}_{*}^{i} \cdot \hat{\boldsymbol{q}}_{h}^{i} d \Omega$.

Using the vectorial notation introduced in $(27) \hat{\boldsymbol{q}}_{*}^{i}=\boldsymbol{Q} \boldsymbol{c}$, the local complementary energy is

$$
\begin{aligned}
\int_{\omega_{i}} \hat{\boldsymbol{q}}^{i} \cdot \hat{\boldsymbol{q}}^{i} d \Omega & =\int_{\omega_{i}}(\boldsymbol{Q} \boldsymbol{c}) \cdot(\boldsymbol{Q} \boldsymbol{c}) d \Omega-2 \int_{\omega_{i}}(\boldsymbol{Q} \boldsymbol{c}) \cdot \hat{\boldsymbol{q}}_{h}^{i} d \Omega+C^{i} \\
& =\boldsymbol{c}^{\top}\left(\int_{\omega_{i}} \boldsymbol{Q}^{\top} \boldsymbol{Q} d \Omega\right) \boldsymbol{c}-2 \boldsymbol{c}^{\top}\left(\int_{\omega_{i}} \boldsymbol{Q}^{\top} \hat{\boldsymbol{q}}_{h}^{i} d \Omega\right)+C^{i}=: \boldsymbol{c}^{\top} \boldsymbol{M} \boldsymbol{c}-2 \boldsymbol{c}^{\top} \boldsymbol{b}+C^{i}
\end{aligned}
$$

where $C^{i}$ does not depend on $\boldsymbol{c}$.

The minimum of the local complementary energy restricted to the equilibrium conditions (29), $\boldsymbol{D} \boldsymbol{c}=\boldsymbol{d}$, is enforced by the Lagrange multiplier technique

$$
\min _{\boldsymbol{c} \in \mathbb{R}^{2 m N}, \boldsymbol{\lambda} \in \mathbb{R}^{n_{r e s t}}} \boldsymbol{c}^{\top} \boldsymbol{M} \boldsymbol{c}-2 \boldsymbol{c}^{\top} \boldsymbol{b}-2 \boldsymbol{\lambda}^{\top}(\boldsymbol{D} \boldsymbol{c}-\boldsymbol{d})
$$

which yields the linear system of equations

$$
\left(\begin{array}{cc}
M & D^{\top} \\
D & 0
\end{array}\right)\left(\begin{array}{l}
c \\
\lambda
\end{array}\right)=\left(\begin{array}{l}
b \\
d
\end{array}\right)
$$

The matrix $M$ is a symmetric block diagonal matrix, the usual mass matrix. Indeed, if the matrix $\boldsymbol{Q}$ is represented using the matrices $\boldsymbol{Q}_{k}$ defined in the elements of the star

$$
\boldsymbol{Q}=\left[\boldsymbol{Q}_{k_{1}} \boldsymbol{Q}_{k_{2}} \ldots \boldsymbol{Q}_{k_{m}}\right]
$$

yields

$$
\boldsymbol{Q}^{\top} \boldsymbol{Q}=\left(\begin{array}{cccc}
\boldsymbol{Q}_{k_{1}}^{\top} \boldsymbol{Q}_{k_{1}} & \boldsymbol{Q}_{k_{1}}^{\top} \boldsymbol{Q}_{k_{2}} & \ldots & \boldsymbol{Q}_{k_{k}}^{\top} \boldsymbol{Q}_{k_{m}} \\
\boldsymbol{Q}_{k_{2}}^{\top} \boldsymbol{Q}_{k_{1}} & \boldsymbol{Q}_{k_{2}}^{\top} \boldsymbol{Q}_{k_{2}} & \ldots & \boldsymbol{Q}_{k_{2}}^{\top} \boldsymbol{Q}_{k_{m}} \\
\vdots & \vdots & \ddots & \vdots \\
\boldsymbol{Q}_{k_{m}}^{\top} \boldsymbol{Q}_{k_{1}} & \boldsymbol{Q}_{k_{1}}^{\top} \boldsymbol{Q}_{k_{m}} & \ldots & \boldsymbol{Q}_{k_{m}}^{\top} \boldsymbol{Q}_{k_{m}}
\end{array}\right)
$$

Thus, the matrix $\boldsymbol{M}$ is the diagonal block matrix

$$
\boldsymbol{M}=\int_{\omega_{i}} \boldsymbol{Q}^{\top} \boldsymbol{Q} d \Omega=\left(\begin{array}{cccc}
\int_{\Omega_{k_{1}}} \boldsymbol{Q}_{k_{1}}^{\top} \boldsymbol{Q}_{k_{1}} d \Omega & 0 & \ldots & \mathbf{0} \\
\mathbf{0} & \int_{\Omega_{k_{2}}} \boldsymbol{Q}_{k_{2}}^{\top} \boldsymbol{Q}_{k_{2}} d \Omega & \ldots & \mathbf{0} \\
\vdots & \vdots & \ddots & \vdots \\
\mathbf{0} & \mathbf{0} & \ldots & \int_{\Omega_{k_{m}}} \boldsymbol{Q}_{k_{m}}^{\top} \boldsymbol{Q}_{k_{m}} d \Omega
\end{array}\right) .
$$


Moreover, if the flux field $\hat{\boldsymbol{q}}_{h}^{i}$ is represented using the matrix $\boldsymbol{Q}$,

$$
\hat{\boldsymbol{q}}_{h}^{i}=\boldsymbol{Q} \boldsymbol{c}_{h},
$$

then, the vector $\boldsymbol{b}$ is computed using the matrix $\boldsymbol{M}$ as

$$
\boldsymbol{b}:=\int_{\omega_{i}} \boldsymbol{Q}^{\top} \hat{\boldsymbol{q}}_{h}^{i} d \Omega=\left(\int_{\omega_{i}} \boldsymbol{Q}^{\top} \boldsymbol{Q} d \Omega\right) \boldsymbol{c}_{h}=\boldsymbol{M} \boldsymbol{c}_{h} .
$$

5.6.2. Minimization of the accumulated local complementary energy Given a star $\omega_{i}$, the previously computed local estimates $\hat{\boldsymbol{q}}^{j}, j=1, \ldots, i-1$ sum up to build the accumulated global estimate

$$
\hat{\boldsymbol{q}}_{a c}^{i-1}=\sum_{j=1}^{i-1} \hat{\boldsymbol{q}}^{j} .
$$

Then, the local estimates $\hat{\boldsymbol{q}}^{i}$ are chosen such that the accumulated local complementary energy is minimum

$$
\int_{\omega_{i}}\left(\hat{\boldsymbol{q}}_{a c}^{i-1}+\hat{\boldsymbol{q}}^{i}\right) \cdot\left(\hat{\boldsymbol{q}}_{a c}^{i-1}+\hat{\boldsymbol{q}}^{i}\right) d \Omega .
$$

Thus, the goal is to minimize

$$
\begin{aligned}
\int_{\omega_{i}}\left(\hat{\boldsymbol{q}}_{a c}^{i-1}+\hat{\boldsymbol{q}}^{i}\right) \cdot\left(\hat{\boldsymbol{q}}_{a c}^{i-1}+\hat{\boldsymbol{q}}^{i}\right) d \Omega & =\int_{\omega_{i}}\left(\hat{\boldsymbol{q}}_{a c}^{i-1}+\hat{\boldsymbol{q}}_{*}^{i}-\hat{\boldsymbol{q}}_{h}^{i}\right) \cdot\left(\hat{\boldsymbol{q}}_{a c}^{i-1}+\hat{\boldsymbol{q}}_{*}^{i}-\hat{\boldsymbol{q}}_{h}^{i}\right) d \Omega \\
& =\int_{\omega_{i}}\left(\hat{\boldsymbol{q}}_{*}^{i}-\left(\hat{\boldsymbol{q}}_{h}^{i}-\hat{\boldsymbol{q}}_{a c}^{i-1}\right)\right) \cdot\left(\hat{\boldsymbol{q}}_{*}^{i}-\left(\hat{\boldsymbol{q}}_{h}^{i}-\hat{\boldsymbol{q}}_{a c}^{i-1}\right)\right) d \Omega
\end{aligned}
$$

As in the previous approach it is useful to represent the flux field $\hat{\boldsymbol{q}}^{i-1}$ using the matrix $\boldsymbol{Q}$,

$$
\hat{\boldsymbol{q}}_{a c}^{i-1}=\boldsymbol{Q} \boldsymbol{c}_{a c},
$$

which is associated with the vector $\boldsymbol{b}_{a c}$

$$
\boldsymbol{b}_{a c}:=\int_{\omega_{i}} \boldsymbol{Q}^{\top} \hat{\boldsymbol{q}}_{a c}^{i-1} d \Omega=\boldsymbol{M} \boldsymbol{c}_{a c}
$$

In this case, the minimization problem that has to be solved is

$$
\min _{\boldsymbol{c} \in \mathbb{R}^{2 m N}, \boldsymbol{\lambda} \in \mathbb{R}^{n_{r e s t}}} \boldsymbol{c}^{\top} \boldsymbol{M c}-2 \boldsymbol{c}^{\top}\left(\boldsymbol{b}-\boldsymbol{b}_{a c}\right)-2 \boldsymbol{\lambda}^{\top}(\boldsymbol{D} \boldsymbol{c}-\boldsymbol{d})
$$

which yields the linear system of equations to determine the unknowns $\boldsymbol{c}$

$$
\left(\begin{array}{cc}
M & D^{\top} \\
D & 0
\end{array}\right)\left(\begin{array}{c}
c \\
\lambda
\end{array}\right)=\left(\begin{array}{c}
b-b_{a c} \\
d
\end{array}\right)
$$

where the only difference with the previous approach is that the r.h.s. term of the equations concerning the minimization of the local complementary energy contains an extra term.

\section{ALTERNATIVE REPRESENTATION FOR $\hat{\boldsymbol{q}}_{*}^{i}$}

An alternative representation of the flux fields $\hat{\boldsymbol{q}}_{*}^{i}$ is considered by taking advantage of the decomposition of the space $\mathcal{H}\left(\operatorname{div}, \Omega_{k}\right)$ used in the context of mixed or hybrid elements.

\subsection{Piecewise polynomial representation for $\left.\hat{\boldsymbol{q}}_{*}^{i}\right|_{\Omega_{k}}$}

Let $\boldsymbol{q}_{k}$ be a flux field in an element $\Omega_{k}$ such that $\boldsymbol{q}_{k} \in\left[\mathbb{P}^{q}\left(\Omega_{k}\right)\right]^{2}$, that is, each component of the flux field $\boldsymbol{q}_{k}$ is a polynomial of degree $q$ inside the element $\Omega_{k}$. In this case, the flux field can be represented as

$$
\boldsymbol{q}_{k}=\boldsymbol{Q}_{k} \boldsymbol{c}_{k}
$$


where $\boldsymbol{Q}_{k}$ and $\boldsymbol{c}_{k}$ are defined in equation (26). Recall that the total number of unknowns in $2 \mathrm{D}$ is $2 N=(q+1)(q+2)$.

However, this is not the natural representation of the flux field $\boldsymbol{q}_{k}$ to enforce equations (24). In order to introduce the natural degrees of freedom, some functional spaces have to be introduced. Let $\mathrm{R}^{q}\left(\partial \Omega_{k}\right)$ be the space of functions which are polynomial of degree $q$ along the edges of the triangle $\partial \Omega_{k}$ and let $\mathbb{P}_{0}^{q}\left(\Omega_{k}\right)$ be the space of polynomials of degree $q$ in $\Omega_{k}$ with zero average, that is:

$$
\mathbb{P}_{0}^{q}\left(\Omega_{k}\right)=\left\{v \in \mathbb{P}^{q}\left(\Omega_{k}\right), \int_{\Omega_{k}} v=0\right\}
$$

Let us consider the space

$$
\Phi^{q}\left(\Omega^{k}\right)=\left\{\boldsymbol{p}_{k} \in\left[\mathbb{P}^{q}\left(\Omega_{k}\right)\right]^{2}, \boldsymbol{\nabla} \cdot \boldsymbol{p}_{k}=0 \text { in } \Omega_{k}, \boldsymbol{p}_{k} \cdot \boldsymbol{n}_{k}=0 \text { on } \partial \Omega_{k}\right\},
$$

where $\boldsymbol{n}_{k}$ denotes the unit outward normal to the element $\Omega_{k}$. In fact, the space $\Phi^{q}\left(\Omega^{k}\right)$ is also described using the cubic bubble function $b_{k}$ vanishing on $\partial \Omega_{k}$ as

$$
\Phi^{q}\left(\Omega^{k}\right)=\left\{\operatorname{curl}\left(b_{k} v\right), v \in \mathbb{P}^{q-2}\left(\Omega_{k}\right)\right\} .
$$

Then, the natural degrees of freedom for $\boldsymbol{q}_{k}$ are the following:

$$
\begin{array}{ll}
\int_{\Omega_{k}} \boldsymbol{q}_{k} \cdot \nabla v d \Omega & \forall v \in \mathbb{P}_{0}^{q-1}\left(\Omega_{k}\right), \\
\int_{\partial \Omega_{k}}^{\boldsymbol{q}_{k} \cdot \boldsymbol{n}_{k} v d \Omega} & \forall v \in \mathrm{R}^{q}\left(\partial \Omega_{k}\right), \\
\int_{\Omega_{k}}^{\boldsymbol{q}_{k} \cdot \boldsymbol{p}_{k} d \Omega} & \forall \boldsymbol{p}_{k} \in \Phi^{q}\left(\Omega^{k}\right) .
\end{array}
$$

It is worth noting that $\operatorname{dim}\left(\mathbb{P}_{0}^{q-1}\left(\Omega_{k}\right)\right)=q(q+1) / 2-1, \operatorname{dim}\left(\mathrm{R}^{q}\left(\partial \Omega_{k}\right)\right)=3(q+1)$ and $\operatorname{dim}\left(\Phi^{q}\left(\Omega^{k}\right)\right)=\operatorname{dim}\left(\mathbb{P}^{q-2}\left(\Omega_{k}\right)\right)=(q-1) q / 2$, thus

$$
\operatorname{dim}\left(\mathbb{P}_{0}^{q-1}\left(\Omega_{k}\right)\right)+\operatorname{dim}\left(\mathrm{R}^{q}\left(\partial \Omega_{k}\right)\right)+\operatorname{dim}\left(\Phi^{q}\left(\Omega^{k}\right)\right)=(q+1)(q+2)=2 N .
$$

\subsection{Statically admissible flux field $\hat{\boldsymbol{q}}_{*}^{i}$}

In order to obtain a statically admissible flux field, $\hat{\boldsymbol{q}}_{*}^{i}$ has to verify the restrictions given by $(24)$.

The degrees of freedom of $\hat{\boldsymbol{q}}_{*}^{i}$ in the star $\omega_{i}$ composed by the elements of indices $K_{i}=$ $\left\{k_{1}, k_{2}, \ldots, k_{m}\right\}$ are for $j=1, \ldots, m$

$$
\begin{aligned}
& \int_{\Omega_{k_{j}}} \boldsymbol{q}_{k_{j}} \cdot \nabla v d \Omega \quad \forall v \in \mathbb{P}_{0}^{q-1}\left(\Omega_{k_{j}}\right), \\
& \int_{\partial \Omega_{k_{j}}} \boldsymbol{q}_{k_{j}} \cdot \boldsymbol{n}_{k_{j}} v d \Omega \forall v \in \mathrm{R}^{q}\left(\partial \Omega_{k_{j}}\right), \\
& \int_{\Omega_{k_{j}}} \boldsymbol{q}_{k_{j}} \cdot \boldsymbol{p}_{k} d \Omega \quad \forall \boldsymbol{p}_{k_{j}} \in \Phi^{q}\left(\Omega^{k_{j}}\right),
\end{aligned}
$$

where $\boldsymbol{q}_{k_{j}}$ represents the restriction of the flux field $\hat{\boldsymbol{q}}_{*}^{i}$ to the element $\Omega_{k_{j}}$.

Using these degrees of freedom, equations (24b) affect only the degrees of freedom associated with (30b). Indeed, equations (24b) yield

$$
\int_{\gamma} \boldsymbol{q}_{k_{j}} \cdot \boldsymbol{n}_{k_{j}} v d \Omega=0 \quad \forall v \in \mathrm{R}^{q}(\gamma), \gamma \in \partial \Omega_{k_{j}} \cap \partial \omega_{i}-\left\{\Gamma_{\mathrm{N}} \cup \Gamma_{\mathrm{D}}\right\} .
$$

Similarly, equations (24c) yield

$$
\int_{\gamma} \boldsymbol{q}_{k_{j}} \cdot \boldsymbol{n}_{k_{j}} v d \Omega=\int_{\gamma} \phi_{i} g v d \Omega \quad \forall v \in \mathrm{R}^{q}(\gamma), \gamma \in \partial \Omega_{k_{j}} \cap \Gamma_{\mathrm{N}} .
$$


Finally, equations (24d) also affect the degrees of freedom associated with (30b), since they yield

$$
\int_{\gamma} \boldsymbol{q}_{k_{j}} \cdot \boldsymbol{n}_{k_{j}} v d \Omega+\int_{\gamma} \boldsymbol{q}_{k_{l}} \cdot \boldsymbol{n}_{k_{l}} v d \Omega=0 \quad \forall v \in \mathrm{R}^{q}(\gamma), \gamma \in \partial \Omega_{k_{j}} \cap \partial \Omega_{k_{l}} .
$$

Thus, equations (24b), (24c) and (24d) only affect the degrees of freedom associated with (30b).

Using these degrees of freedom, it is also easy to impose (24a) and in this case these restrictions relate both the degrees of freedom associated with (30a) and (30b). Indeed, equation (24a) is equivalent to imposing

$$
-\boldsymbol{\nabla} \cdot \boldsymbol{q}_{k_{j}}=\phi_{i} f-\boldsymbol{\nabla} \phi_{i} \cdot \nabla u_{H} \quad \text { in } \Omega_{k_{j}}, j=1, \ldots, m .
$$

Since all the expressions in the previous equations are at most polynomials of degree $q-1$, the previous equation is equivalent to

$$
\int_{\Omega_{k_{j}}}-\nabla \cdot \boldsymbol{q}_{k_{j}} v d \Omega=\int_{\Omega_{k_{j}}}\left[\phi_{i} f-\nabla \phi_{i} \cdot \nabla u_{H}\right] v d \Omega \quad \forall v \in \mathbb{P}_{0}^{q-1}\left(\Omega_{k_{j}}\right) \cup \mathbb{P}^{0}\left(\Omega_{k_{j}}\right) .
$$

Now, integrating by parts the l.h.s. yields

$\int_{\Omega_{k_{j}}}-\boldsymbol{\nabla} \cdot \boldsymbol{q}_{k_{j}} v d \Omega=\int_{\Omega_{k_{j}}}\left[-\boldsymbol{\nabla} \cdot\left(\boldsymbol{q}_{k_{j}} v\right)+\boldsymbol{q}_{k_{j}} \cdot \boldsymbol{\nabla} v\right] d \Omega=\int_{\Omega_{k_{j}}} \boldsymbol{q}_{k_{j}} \cdot \boldsymbol{\nabla} v d \Omega-\int_{\partial \Omega_{k_{j}}} \boldsymbol{q}_{k_{j}} \cdot \boldsymbol{n}_{k_{j}} v d \Gamma$.

Thus, equation (24a) is equivalent to

$\int_{\Omega_{k_{j}}} \boldsymbol{q}_{k_{j}} \cdot \boldsymbol{\nabla} v d \Omega-\int_{\partial \Omega_{k_{j}}} \boldsymbol{q}_{k_{j}} \cdot \boldsymbol{n}_{k_{j}} v d \Gamma=\int_{\Omega_{k_{j}}}\left[\phi_{i} f-\boldsymbol{\nabla} \phi_{i} \cdot \boldsymbol{\nabla} u_{H}\right] v d \Omega \quad \forall v \in \mathbb{P}_{0}^{q-1}\left(\Omega_{k_{j}}\right) \cup \mathbb{P}^{0}\left(\Omega_{k_{j}}\right)$.

For $v \in \mathbb{P}^{0}\left(\Omega_{k_{j}}\right)$, that is, for $v$ constant in the element $\Omega_{k_{j}}$, the previous restriction affects the degrees of freedom associated with $(30 \mathrm{~b})$

$$
-\int_{\partial \Omega_{k_{j}}} \boldsymbol{q}_{k_{j}} \cdot \boldsymbol{n}_{k_{j}} d \Gamma=\int_{\Omega_{k_{j}}}\left[\phi_{i} f-\nabla \phi_{i} \cdot \nabla u_{H}\right] d \Omega,
$$

whereas $v \in \mathbb{P}_{0}^{q-1}\left(\Omega_{k_{j}}\right)$ determines the degrees of freedom associated with (30a) in terms of the degrees of freedom on the boundary associated with (30b),

$$
\int_{\Omega_{k_{j}}} \boldsymbol{q}_{k_{j}} \cdot \boldsymbol{\nabla} v d \Omega=\int_{\partial \Omega_{k_{j}}} \boldsymbol{q}_{k_{j}} \cdot \boldsymbol{n}_{k_{j}} v d \Gamma+\int_{\Omega_{k_{j}}}\left[\phi_{i} f-\boldsymbol{\nabla} \phi_{i} \cdot \nabla u_{H}\right] v d \Omega \quad \forall v \in \mathbb{P}_{0}^{q-1}\left(\Omega_{k_{j}}\right) .
$$

Once the degrees of freedom on the boundary of the elements (30b) are set, equation (35) determines the value for the interior degrees of freedom (30a). Moreover, it is clear that the degrees of freedom associated with (30c) may be set arbitrarily since they do not affect the conditions of being statically admissible.

Equations (31), (32), (33) and (34) are $\left(m_{b}+m_{i}\right)(q+1)+m$ restrictions for the degrees of freedom associated with the boundary, (30b). However, one of these equations is linearly dependent of the others in the case of a star that does not intersect the Dirichlet boundary. On the other hand, equations (35) uniquely determine the degrees of freedom related to the interior, (30a).

Thus, for a star that does not intersect the Dirichlet boundary, the degrees of freedom which are not determined by the condition of being statically admissible are the degrees of freedom lying on the boundary not determined by (31), (32), (33) and (34) and the degrees of freedom associated with the space $\Phi^{q}\left(\Omega^{k_{j}}\right)$. That is, there are

$$
3 m(q+1)-\left(m_{b}+m_{i}\right)(q+1)-m-1+m(q-1) q / 2
$$

degrees of freedom which may be used to minimize the complementary energy. 


\subsection{Complementary energy minimization}

The use of the natural degrees of freedom (30) shows that given a statically admissible flux field $\hat{\boldsymbol{q}}$, then

$$
\hat{\boldsymbol{q}}+\hat{\boldsymbol{q}}_{\Phi}
$$

is also a statically admissible flux field, where $\hat{\boldsymbol{q}}_{\Phi} \in \widehat{\Phi}^{q}(\Omega)$,

$$
\widehat{\Phi}^{q}(\Omega)=\left\{\hat{\boldsymbol{q}},\left.\hat{\boldsymbol{q}}\right|_{\Omega_{k}} \in \Phi^{q}\left(\Omega_{k}\right)\right\}
$$

Thus, given a computed statically admissible flux field a simple post-process in each element of the mesh allows minimizing the total complementary energy. Given a statically admissible flux field $\hat{\boldsymbol{q}}$, the goal is to compute $\hat{\boldsymbol{q}}_{\Phi} \in \widehat{\Phi}^{q}(\Omega)$ minimizing

$$
\int_{\Omega}\left(\hat{\boldsymbol{q}}+\hat{\boldsymbol{q}}_{\Phi}\right) \cdot\left(\hat{\boldsymbol{q}}+\hat{\boldsymbol{q}}_{\Phi}\right) d \Omega=\sum_{k=1}^{n_{\mathrm{el}}} \int_{\Omega_{k}}\left(\boldsymbol{q}_{k}+\boldsymbol{q}_{\Phi k}\right) \cdot\left(\boldsymbol{q}_{k}+\boldsymbol{q}_{\Phi k}\right) d \Omega
$$

where $\boldsymbol{q}_{k}$ and $\boldsymbol{q}_{\Phi k}$ are the restrictions of the global flux fields $\hat{\boldsymbol{q}}$ and $\hat{\boldsymbol{q}}_{\Phi}$ to the element $\Omega_{k}$. Moreover, $\boldsymbol{q}_{\Phi k}=\operatorname{curl}\left(b_{k} v\right)$ for some $v \in \mathbb{P}^{q-2}\left(\Omega_{k}\right)$.

Thus, the minimization of the total energy (36) yields $n_{\mathrm{el}}$ independent minimization problems, one for each element of the mesh,

$$
\min \int_{\Omega_{k}}\left(\boldsymbol{q}_{k}+\boldsymbol{q}_{\Phi k}\right) \cdot\left(\boldsymbol{q}_{k}+\boldsymbol{q}_{\Phi k}\right) d \Omega
$$

Since both $\boldsymbol{q}_{k}$ and $\boldsymbol{q}_{\Phi k}$ belong to the space $\left[\mathbb{P}^{q}\left(\Omega_{k}\right)\right]^{2}$, using the vectorial notation introduced in (26), the local flux fields $\boldsymbol{q}_{k}$ and $\boldsymbol{q}_{\Phi k}$ is represented using the matrix $\boldsymbol{Q}_{k}$ as

$$
\boldsymbol{q}_{k}=\boldsymbol{Q}_{k} \boldsymbol{c}_{k} \quad, \quad \boldsymbol{q}_{\Phi k}=\boldsymbol{Q}_{k} \boldsymbol{c}_{\Phi k}
$$

and the complementary energy in the element $\Omega_{k}$ is

$$
\begin{aligned}
\int_{\Omega_{k}}\left(\boldsymbol{q}_{k}+\boldsymbol{q}_{\Phi k}\right) \cdot\left(\boldsymbol{q}_{k}+\boldsymbol{q}_{\Phi k}\right) d \Omega & =\int_{\Omega_{k}} \boldsymbol{q}_{k} \cdot \boldsymbol{q}_{k} d \Omega+\int_{\Omega_{k}} \boldsymbol{q}_{\Phi k} \cdot \boldsymbol{q}_{\Phi k} d \Omega+2 \int_{\Omega_{k}} \boldsymbol{q}_{k} \cdot \boldsymbol{q}_{\Phi k} d \Omega \\
& =\boldsymbol{c}_{\Phi k}^{\top}\left(\int_{\Omega_{k}} \boldsymbol{Q}_{k}^{\top} \boldsymbol{Q}_{k} d \Omega\right) \boldsymbol{c}_{\Phi k}+2 \boldsymbol{c}_{\Phi k}^{\top}\left(\int_{\Omega_{k}} \boldsymbol{Q}_{k}^{\top} \boldsymbol{Q}_{k} d \Omega\right) \boldsymbol{c}_{k}+C^{k} \\
& =\boldsymbol{c}_{\Phi k}^{\top} \boldsymbol{M}_{k} \boldsymbol{c}_{\Phi k}+2 \boldsymbol{c}_{\Phi k}^{\top} \boldsymbol{M}_{k} \boldsymbol{c}_{k}+C^{k}
\end{aligned}
$$

where $C^{k}$ does not depend on the degrees of freedom $\boldsymbol{c}_{\Phi k}$.

Note however that not all the values $\boldsymbol{c}_{\Phi k}$ are actual degrees of freedom since $\operatorname{dim}\left(\Phi^{q}\left(\Omega^{k}\right)\right)=$ $(q-1) q / 2$ while $\boldsymbol{c}_{\Phi k} \in \mathbb{R}^{(q+1)(q+2)}$. Then, denoting by $\boldsymbol{c}_{\Phi k}^{\text {dof }}$ the actual degrees of freedom, one has

$$
\boldsymbol{c}_{\Phi k}=\boldsymbol{Q}_{\Phi} \boldsymbol{c}_{\Phi k}^{\mathrm{dof}}
$$

where $\boldsymbol{Q}_{\Phi}$ is the $(q+1)(q+2) \times(q-1) q / 2$ transformation matrix.

Thus, the minimization problem reads:

$$
\min _{\boldsymbol{c}_{\Phi k}^{\mathrm{dof}} \in \mathbb{R}^{(q-1) q / 2}}\left(\boldsymbol{c}_{\Phi k}^{\mathrm{dof}}\right)^{\top} \boldsymbol{Q}_{\Phi}^{\top} \boldsymbol{M}_{k} \boldsymbol{Q}_{\Phi} \boldsymbol{c}_{\Phi k}^{\mathrm{dof}}+2\left(\boldsymbol{c}_{\Phi k}^{\mathrm{dof}}\right)^{\top} \boldsymbol{Q}_{\Phi}^{\top} \boldsymbol{M}_{k} \boldsymbol{c}_{k}+C^{k}
$$

leading to the system of equations

$$
\boldsymbol{Q}_{\Phi}^{\top} \boldsymbol{M}_{k} \boldsymbol{Q}_{\Phi} \boldsymbol{c}_{\Phi k}^{\mathrm{dof}}=-\boldsymbol{Q}_{\Phi}^{\top} \boldsymbol{M}_{k} \boldsymbol{c}_{k}
$$

Note that for $q=2, \Phi^{2}\left(\Omega^{k}\right)=\left\{\operatorname{curl}\left(b_{k} v\right), v \in \mathbb{P}^{0}\left(\Omega_{k}\right)\right\}=\left\langle\operatorname{curl}\left(b_{k}\right)\right\rangle$. 


\subsection{Non-polynomial source terms or Neumann boundary conditions}

The strategy presented so far is only applicable to problems where the source term $f$ and Neumann boundary conditions $g$ are piecewise polynomial. However, using the strategies presented in $[17,27]$ this restriction on $f$ and $g$ could be removed.

In [17] a strategy to obtain strict upper bounds for the energy norm of the diffusion-reaction equation is proposed. The strategy follows the same rationale as the strategy presented in [11] but allows computing strict bounds for non-polynomial source terms. The difference between the two approaches is the way to find the locally admissible flux field in the elements of the coarse mesh. Although the strategy presented in [17] is more general, it is more complex and less natural to implement. Thus, it would be advisable to use the local construction in [17] only in those elements where the source term or boundary conditions are non polynomial.

In the context of the flux-free estimates, the approach presented in [17] could also be used to yield strict bounds even in the problems where the source term and Neumann boundary conditions are non-polynomials. However, the implementation of this approach is much more complex and it is only needed in those stars where the data is non-polynomial.

\subsection{Computational cost}

This section is devoted to compare the computational effort required to solve the local problems for the guaranteed bounds, with respect to the cost of the asymptotic version [18]. In both cases, the cost of computing a strict upper bound for the energy norm of the error is proportional to the number of vertex nodes in the mesh. Once the finite element approximation $u_{H}$ is computed, a local problem for each star is solved with a constant cost that only depends on the interpolation polynomial degree $q$ of the dual estimates $\hat{\boldsymbol{q}}_{*}^{i}$ (or in the local refinement parameter in the asymptotic version).

Consider an interior star $\omega_{i}$ thus not intersecting the boundary of the domain. In order to obtain an upper bound for the reference error $\left\|e_{h}\right\|$, which in turn provides an asymptotic upper bound for $\|e\|$, the local problems (8) have to be solved in a local reference mesh, see figure (4). The cost of solving the local problems depends on the local refinement parameter.
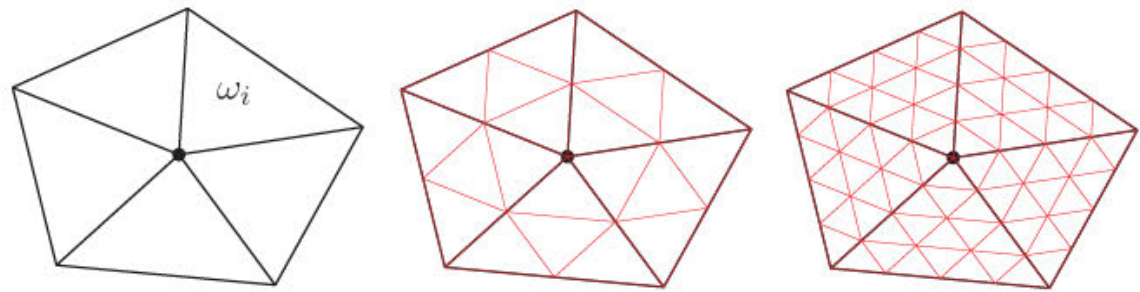

Figure 4. Local reference mesh in an interior star $\omega_{i}$ with $m=5$ elements for different local refinement parameters: $n_{h}=2$ in the center and $n_{h}=4$ in the right.

Let $n_{h}$ be the refinement of each edge of the triangles in a star, that is, each triangle is divided into $n_{h}^{2}$ new triangles. In this case, the local estimate $e_{\omega_{i}}$ depends on $\frac{1}{2}\left(n_{h}+1\right) n_{h} m+1$ degrees of freedom which are determined from (8) solving a linear system of equations.

Strict upper bounds for $\|e\|$ are computed from the dual estimates $\hat{\boldsymbol{q}}_{*}^{i}$ verifying problem (24). The number of degrees of freedom in each star is $m(q+1)(q+2)$. The cost of determining $\hat{\boldsymbol{q}}_{*}^{i}$ varies considerably with the technique used to solve the convex quadratic optimization problem restricted to the $\frac{1}{2}(q+4)(q+1) m-1$ linear equations. The procedure introduced in 5 using Lagrange multipliers is selected for the sake of simplicity in the presentation. Nevertheless, it is not the most efficient implementation of the local problems. The cost of the algorithm could be reduced selecting a more efficient strategy to enforce the constraints.

In order to compare the cost of computing asymptotic versus strict bounds, it is worth noting that the choice of the interpolation degree $q$ depends on the input data $f$ and $g$. For instance, for constant source $f$ and linear applied fluxes $g$, is is sufficient to consider $q=2$. Increasing the local interpolation degree in general improves the bounds since the optimization procedure 
has more degrees of freedom. However, numerical examples show that there is no considerable gain in increasing the interpolation degree $q$. On the contrary, the choice of the local refinement parameter $n_{h}$ is crucial in order to obtain upper bounds which indeed overestimate $\|e\|$. Small values of $n_{h}$ the reference error provides a crude approximation of $\|e\|$ and the upper bound for $\left\|e_{h}\right\|$ is not in general an upper bound for $\|e\|$.

Table I provides a brief sketch of the degrees of freedom for both the asymptotic and the strict strategies. As can be seen, the cost of computing the dual estimates $\hat{\boldsymbol{q}}_{*}^{i}$ for $q=2$ using the less efficient strategy (Lagrange multipliers) is the same as the asymptotic bounds for $n_{h}=6$.

\begin{tabular}{c|ccccc}
\multicolumn{6}{c}{ asymptotic bounds } \\
\hline$n_{h}$ & 2 & 3 & 4 & 5 & 6 \\
\hline d.o.f. & $3 m+1$ & $6 m+1$ & $10 m+1$ & $15 m+1$ & $21 m+1$ \\
\hline \multicolumn{5}{c}{ strict bounds } \\
\hline \multicolumn{7}{c}{ free d.o.f. } & d.o.f. lagr. mult. \\
\hline$q$ & d.o.f. & $n_{\text {rest }}$ & frec & \\
\hline 2 & $12 m$ & $9 m-1$ & $3 m+1$ & $21 m-1$ \\
\hline 3 & $20 m$ & $14 m-1$ & $6 m+1$ & $34 m-1$ \\
\hline
\end{tabular}

Table I. Number of degrees of freedom of the local problems for an interior star with $m$ elements, both for the asymptotic and strict strategies.

\section{NUMERICAL EXAMPLES}

The behavior of the estimates presented above is analyzed in four numerical examples. Some of the selected examples have been used by other authors to assess the performance of different error estimation techniques for the Poisson problem [11, 28, 29, 30].

Linear triangular finite elements and subproblems of degree $3, q=3$, are employed in all the examples. In the examples where the analytical solution is known, the quality of the error estimates is measured with the index

$$
\rho:=\|e\|_{\text {est }} /\|e\|-1 .
$$

Index $\rho$ is the usual effectivity index minus one. The accuracy of the error estimate is given by the absolute value of $\rho$ and the sign indicates if the estimate is an overestimation (positive $\rho$ ) or an underestimation (negative $\rho$ ) of the true error. For instance, $\rho=2 \%$ indicates that the estimated error is larger than the true error with a factor 1.02 and $\rho=-0.3 \%$ means that the true error is underestimated by a factor 0.97 . Note that for strategies providing strict upper bounds for the energy, $\rho$ is positive, whereas asymptotic strategies may provide negative values of $\rho$ mainly for coarse meshes.

The statically admissible flux field $\hat{\boldsymbol{q}}$ also provides local error information which can be used as an indicator for mesh adaptivity via the elemental contribution to the total complementary energy,

$$
\|e\|_{\mathrm{ub}}^{2}:=\int_{\Omega} \hat{\boldsymbol{q}} \cdot \hat{\boldsymbol{q}} d \Omega=\sum_{k=1}^{n_{\mathrm{el}}} \int_{\Omega_{k}} \hat{\boldsymbol{q}} \cdot \hat{\boldsymbol{q}} d \Omega=: \sum_{k=1}^{n_{\mathrm{el}}} \eta_{k} .
$$

The elemental contributions $\eta_{k}$ can serve as informative mesh adaptivity indicators for controlling the error in the energy norm.

Two different adaptive strategies are used both based on the elemental contributions $\eta_{k}$ to the total upper bound $\|e\|_{\mathrm{ub}}^{2}$. First, at each level of refinement, only elements for which $\eta_{k}>\|e\|_{\text {tol }}^{2} / n_{\mathrm{el}}$ are refined, where $\|e\|_{\text {tol }}^{2}$ is a user specified tolerance for the precision of the global energy norm and $n_{\mathrm{el}}$ is the number of elements in the triangulation at that level. Second, the target $\|e\|_{\mathrm{ub}}^{2}<\|e\|_{\mathrm{tol}}^{2}$ is achieved producing a series of adapted meshes by subdividing at each remeshing step a fixed percentage of the elements, those with larger contribution to the 
upper bound $\|e\|_{\mathrm{ub}}^{2}$. In both strategies, by preferentially refining the mesh elements contributing more to the total upper bound, we obtain a simple but effective adaptive strategy.

The behavior of the upper bound for the energy norm introduced in the present work is compared with the strategies presented in [18, 31, 11]. As mentioned in section 3.3, [18] solves the local problems (8) using a local finer mesh, thus yielding asymptotic upper bounds for the energy norm. The strategy presented in [31] is a classical hybrid flux method where first the (linear) equilibrated fluxes are computed and then the local elementary problems are solved using a local fine submesh (asymptotic upper bound). Finally the results are compared to the strategy presented in [11] which also provides strict bounds for the energy norm and differs from [31] in the solution of the local elementary problems. Instead of using a local submesh, strict bounds for the energy are recovered using a dual approach.

The bounds computed using the strategies presented in [18], [31] and [11] are denoted in the following as asymptotic flux-free bounds, asymptotic equilibrated bounds and strict equilibrated bounds respectively.

\subsection{Uniformly Forced Square Domain}

A simple diffusion model for the temperature distribution $u(x, y)$ in a square plate $\Omega=$ $(-1,1) \times(-1,1)$ is considered. The specific source term in this example models uniform heating of the plate $f=1$, and the boundary condition models the edge of the plate being kept at an ice-cold temperature, that is, homogeneous Dirichlet boundary conditions are considered on all the boundary.

The simple shape of the domain enables the solution to be explicitly represented,

$$
u(x, y)=\frac{1-x^{2}}{2}-\frac{16}{\pi^{3}} \sum_{\text {odd } k=1}^{\infty} \frac{\sin (k \pi(1+x) / 2)(\sinh (k \pi(1+y) / 2)+\sinh (k \pi(1-y) / 2))}{k^{3} \sinh (k \pi)} .
$$
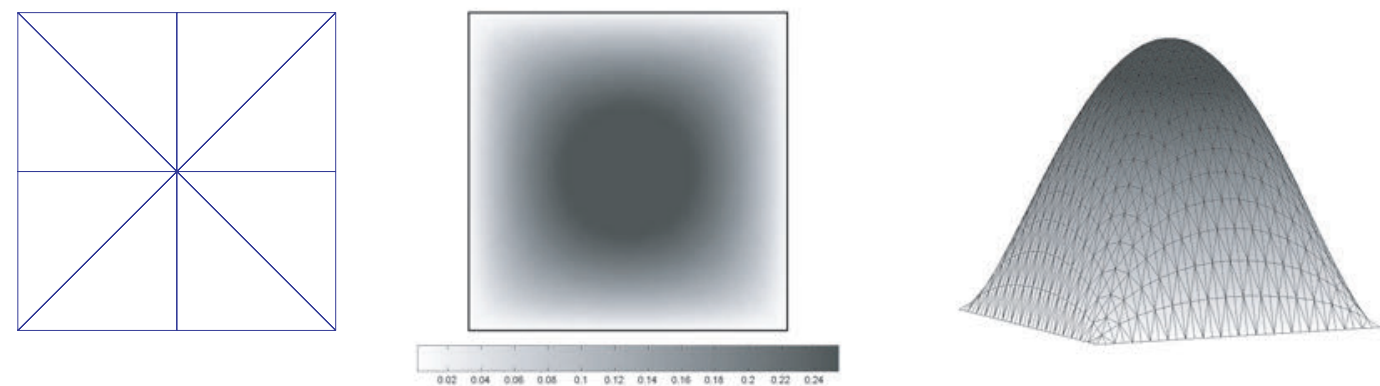

Figure 5. Example 1: series of uniformly $h$-refined linear triangular meshes. Initial mesh (left), finite element approximation on the mesh with 2048 elements (center and right).

The convergence of the bounds is analyzed for a uniform mesh refinement in a series of structured meshes. The initial mesh is composed of 8 triangular elements (half squares) and in each refinement step every triangle is divided into four similar triangles. Both the initial mesh and the finite element approximation obtained with the mesh of 2048 elements are shown in figure 5 .

The upper bounds for the error in the energy norm are shown in table II. Five different strategies have been used to compute an upper bound for $\|e\|$. The upper bounds computed using the flux-free strategy presented in this work - either using the minimization strategy presented in section 5.6.1 or 5.6.2 - are denoted by $\|e\|_{\mathrm{ub}}$ and $\|e\|_{\mathrm{ub}}^{\mathrm{ac}}$ respectively. That is, the degrees of freedom for the flux field $\hat{\boldsymbol{q}}$ are determined either minimizing the local complementary energy or accounting for the accumulated energy. Note that the superscript ac stands for accumulated. The improvement described in section 6.3 has also been implemented for the two approaches (that is, after computing the statically admissible flux field $\hat{\boldsymbol{q}}$, for each element the 3 degrees of freedom associated with $\widehat{\Phi}^{3}(\Omega)$ are determined to minimize the total 


\begin{tabular}{c|cc||ccc|cc}
\hline \multicolumn{2}{l|}{} & \multicolumn{3}{c||}{ strict } & \multicolumn{2}{c}{ asymptotic } \\
\hline$n_{\mathrm{el}}$ & $\left\|u_{H}\right\|$ & $\|e\|$ & $\|e\|_{\mathrm{ub}}$ & $\|e\|_{\mathrm{ub}}^{\mathrm{ac}}$ & $\|e\|_{\mathrm{ub}}^{\mathrm{eq}}$ & $\left\|e_{h}\right\|_{\mathrm{ub}}$ & $\left\|e_{h}\right\|_{\mathrm{ub}}^{\mathrm{eq}}$ \\
\hline 8 & 0.66667 & 0.34331 & 0.34344 & 0.34344 & 0.41276 & 0.32042 & 0.38216 \\
32 & 0.69722 & 0.27604 & 0.28877 & 0.27802 & 0.41079 & 0.27931 & 0.37885 \\
128 & 0.73412 & 0.15288 & 0.15948 & 0.15384 & 0.23194 & 0.15492 & 0.21375 \\
512 & 0.74574 & 0.07857 & 0.08178 & 0.07909 & 0.11949 & 0.07958 & 0.11013 \\
2048 & 0.74883 & 0.03956 & 0.04112 & 0.03984 & 0.06009 & 0.04005 & 0.05539 \\
\hline
\end{tabular}

Table II. Example 1: upper bounds for the error in the energy norm in a series of uniformly $h$-refined linear triangular meshes.

\begin{tabular}{c|c||ccc|cc}
\hline \multicolumn{2}{l||}{} & \multicolumn{3}{c|}{ strict } & \multicolumn{2}{c}{ asymptotic } \\
\hline$n_{\mathrm{el}}$ & $\|e\| /\left\|u_{H}\right\|$ & $\rho$ & $\rho^{\mathrm{ac}}$ & $\rho^{\mathrm{eq}}$ & $\rho_{h}$ & $\rho_{h}^{\mathrm{eq}}$ \\
\hline 8 & 0.51497 & $0.036 \%$ & $0.036 \%$ & $20.228 \%$ & $-6.669 \%$ & $11.316 \%$ \\
32 & 0.39591 & $4.611 \%$ & $0.718 \%$ & $48.817 \%$ & $1.187 \%$ & $37.246 \%$ \\
128 & 0.20825 & $4.314 \%$ & $0.629 \%$ & $51.708 \%$ & $1.333 \%$ & $39.813 \%$ \\
512 & 0.10535 & $4.088 \%$ & $0.671 \%$ & $52.082 \%$ & $1.287 \%$ & $40.172 \%$ \\
2048 & 0.05283 & $3.948 \%$ & $0.698 \%$ & $51.886 \%$ & $1.244 \%$ & $40.017 \%$ \\
\hline
\end{tabular}

Table III. Example 1: quality of the upper bounds, $\rho$, for the error in the energy norm in a series of uniformly $h$-refined linear triangular meshes.

complementary energy). However, the results are nearly identical to those obtained without this minimization strategy and therefore are not shown in table II. Thus, the minimization strategies described in sections 5.6.1 and 5.6.2 implicitly minimize the complementary energy with respect to the degrees of freedom associated with $\widehat{\Phi}^{3}(\Omega)$. The strict upper bound computed using the strategy presented in [11] is denoted by $\|e\|_{\text {ub }}^{\text {eq }}$, where the superscript eq stands for equilibrated. Finally, the asymptotic version of $\|e\|_{\mathrm{ub}}$ and $\|e\|_{\mathrm{ub}}^{\mathrm{eq}}$ have also been computed following [18] and [31] respectively, and are denoted by $\left\|e_{h}\right\|_{\mathrm{ub}}$ and $\left\|e_{h}\right\|_{\mathrm{ub}}^{\mathrm{eq}}$. The reference mesh is obtained dividing each element of the finite element mesh into 16 new elements. Table III shows the quality of the bounds for each of the strategies. As expected, the upper bounds provided by the asymptotic strategies are lower, and hence sharper, than the corresponding upper bounds obtained using strict strategies. However, this is not always a positive feature: for rather coarse meshes the asymptotic upper bound is not an actual upper bound of the exact solution (note the negative sign in the index $\rho_{h}$ ). Since the asymptotic upper bound estimates are only guaranteed to overestimate the reference values, for coarse enough meshes, they do underestimate the exact value $\|e\|$.

It is clear that flux-free strategies provide much better effectivities than the flux equilibration strategies. Moreover, the minimization of the complementary energy accounting for the accumulated nature of the flux field provides very good effectivity indices $\left(\rho^{\text {ac }}<10^{-2}\right.$ which implies an overestimation of the $\|e\|$ of less than $1 \%$ ). In fact, in this example the minimization of the accumulated complementary energy provides better effectivity indices than the asymptotic bounds, i.e. $\rho^{\text {ac }}<\rho_{h}$ in all the meshes but the coarsest one where the index $\rho^{\text {ac }}$ can not be lower than $\rho_{h}$ since strict bounds always produce positive indices $\rho$. Figure 6 also shows the bounds and their effectivity indices. As can be seen, all the strategies have the expected rate of convergence $\mathcal{O}(h)$, and the upper bound $\|e\|_{\text {ub }}^{\text {ac }}$ provides a very good approximation to the exact error $\|e\|$.

Figure 7 shows the spatial distribution of the exact energy norm $\|e\|^{2}$ and of the upper bound $\left(\|e\|_{\mathrm{ub}}^{\mathrm{ac}}\right)^{2}$ for the final mesh of 2048 elements, that is, in each element $\Omega_{k}$ the local contributions $\|e\|_{k}^{2}$ and $\eta_{k}^{\mathrm{ac}}$ are shown. The spatial distribution of $\left(\|e\|_{\mathrm{ub}}^{\mathrm{ac}}\right)^{2}$ is nearly the same as the distribution of $\|e\|^{2}$ thus the quantities $\eta_{k}^{\text {ac }}$ serve as good indicators to drive an adaptive procedure aimed at reducing the error in the energy norm. The spatial distribution of the local effectivity index $\eta_{k}^{\text {ac }} /\|e\|_{k}^{2}$ and the histograms representing the occurrences of local effectivity 

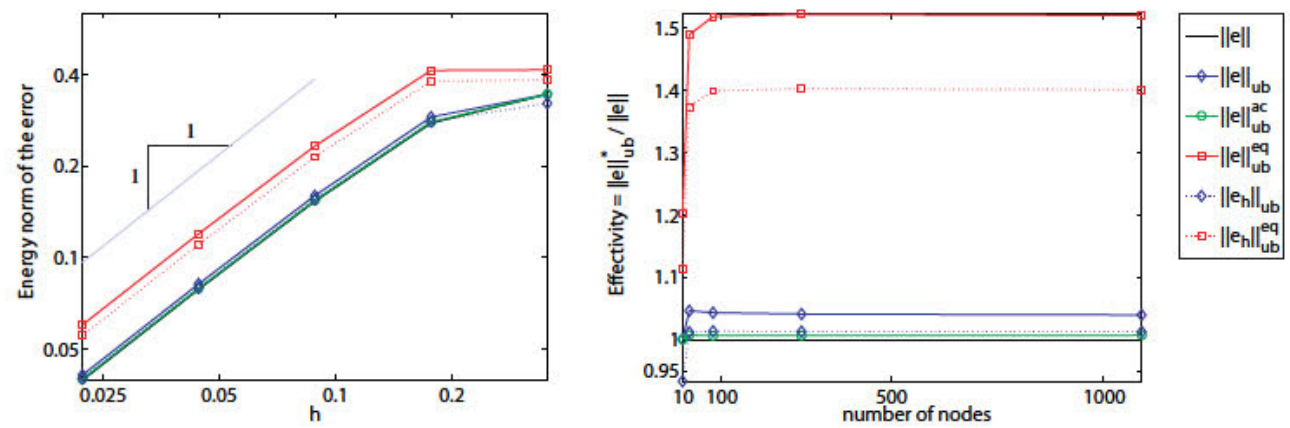

Figure 6. Example 1: series of uniformly $h$-refined linear triangular meshes. Upper bounds for the error in the energy norm (left) and their associated effectivity indices (right).
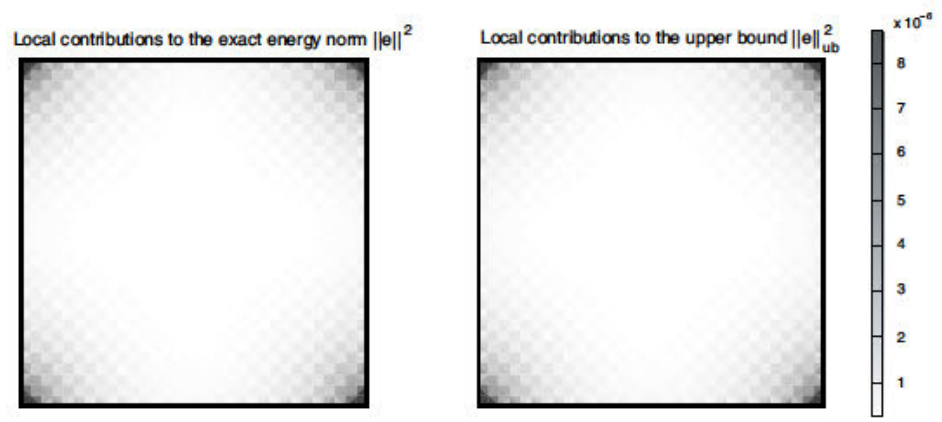

Figure 7. Example 1: spatial distribution of the exact energy norm $\|e\|^{2}$ (left) and of the upper bound $\left(\|e\|_{\mathrm{ub}}^{\mathrm{ac}}\right)^{2}$ (right) for the final mesh of 2048 elements.
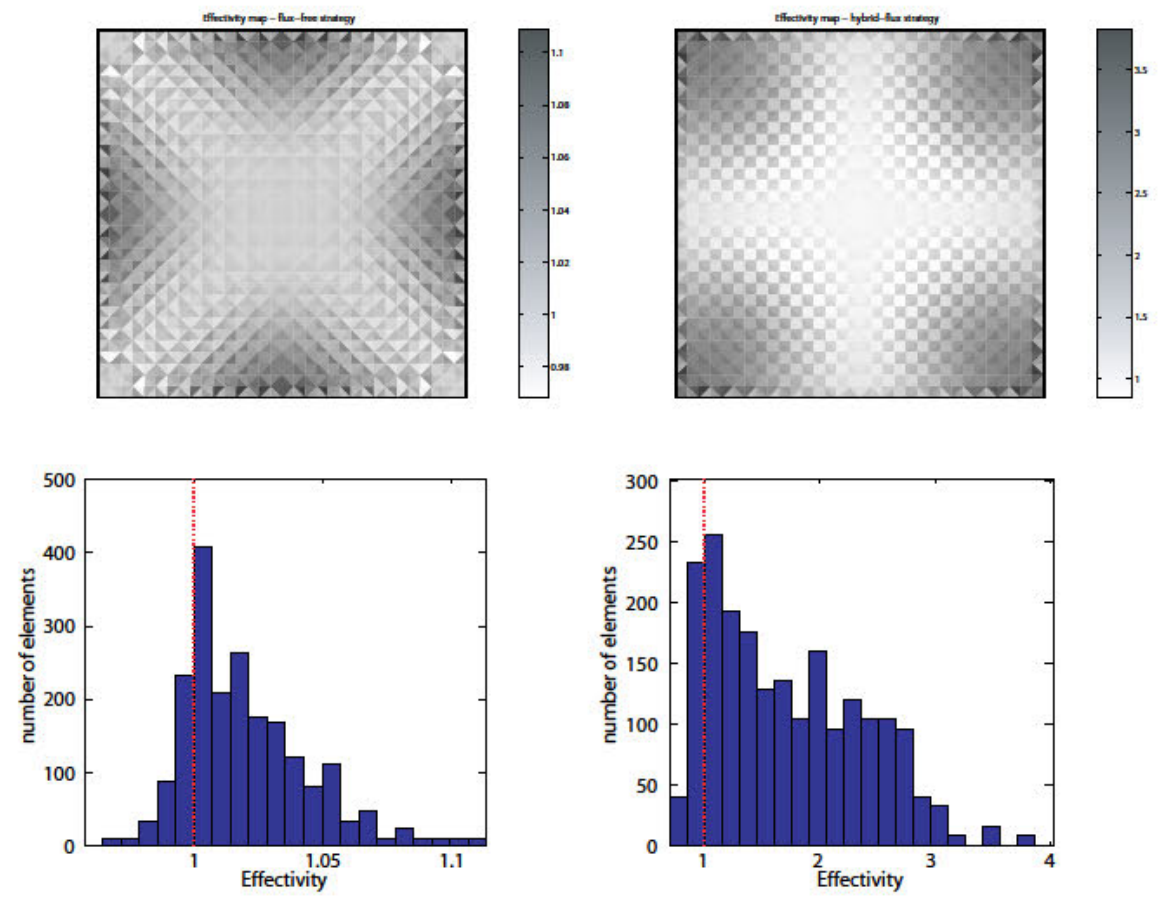

Figure 8. Example 1: spatial distribution of the local effectivity index for the flux-free technique (top-left) and for the hybrid-flux technique (top-right). Histograms for local effectivity indices for the flux-free techniques (down-left) and hybrid-flux techniques (down-right). 
indices are shown in figure 8 for both the estimates $\|e\|_{\mathrm{ub}}^{\mathrm{ac}}$ and $\|e\|_{\mathrm{ub}}^{\mathrm{eq}}$. As can be seen, the flux-free estimate provides much better effectivities than the estimates obtained using hybridfluxes since the local effectivity indices are much more closer to one. The histograms show the number of elements with local effectivity in a given range. The histograms show a good behavior of the estimates if they display a narrow distribution (all elements have similar local effectivity indices) concentrated around 1 . Observe that the local values associated with the estimate $\|e\|_{\mathrm{ub}}^{\mathrm{ac}}$ proposed here are much more accurate than the values corresponding to $\|e\|_{\mathrm{ub}}^{\mathrm{eq}}$.
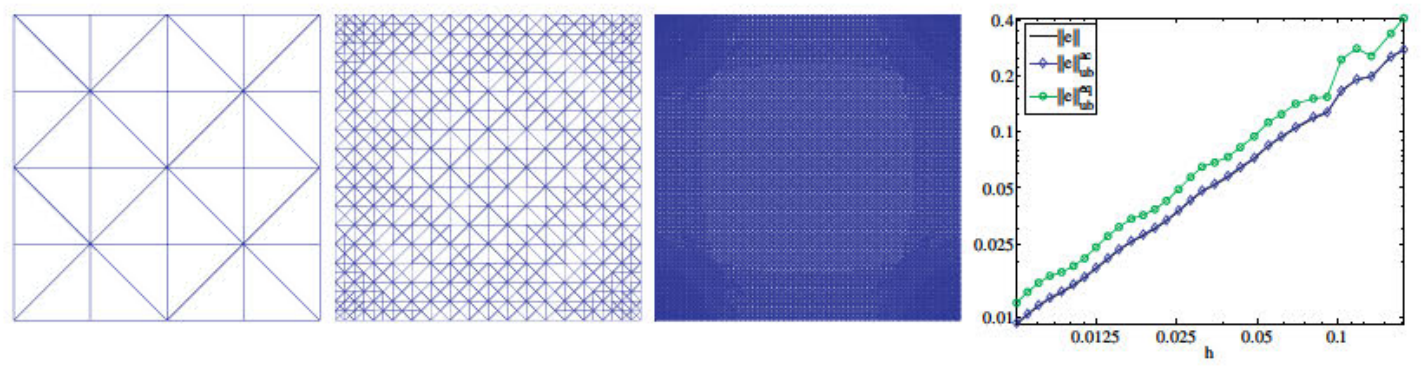

Figure 9. Example 1: series of adaptively $h$-refined linear triangular meshes. Initial, intermediate and final mesh with 32,1032 and 25128 linear elements. In the intermediate mesh $\|e\|_{\mathrm{ub}}^{\mathrm{ac}}<0.05$ and in the final mesh $\|e\|_{\mathrm{ub}}^{\mathrm{ac}}<0.01$. Upper bounds for the error in the energy norm (right).

A series of adapted meshes is produced by subdividing at each remeshing step $20 \%$ of the elements, those with larger contribution to the upper bound of the energy norm, until $\|e\|_{\mathrm{ub}}^{\mathrm{ac}}<0.01$. The adaptive procedure is guided by the indicators $\eta_{k}^{\text {ac }}$ provided by the accumulated strategy proposed in this work. However, in each step, the bounds provided by the strict equilibrated strategy are also computed to compare the results. For an initial mesh of 32 linear elements the actual error is $\|e\|=0.27604$ and the upper bounds are $\|e\|_{\mathrm{ub}}^{\mathrm{ac}}=0.27802(\rho=0.00718)$ and $\|e\|_{\mathrm{ub}}^{\mathrm{eq}}=0.41079(\rho=0.48817)$. After remeshing, the actual error is $\|e\|=0.00926<0.01$ and the upper bounds are $\|e\|_{\mathrm{ub}}^{\mathrm{ac}}=0.00936(\rho=0.01150)$ and $\|e\|_{\mathrm{ub}}^{\mathrm{eq}}=0.01203(\rho=0.29955)$. Figure 9 shows the initial mesh of 32 elements, the final mesh of 25128 elements and the intermediate mesh where $\|e\|_{\mathrm{ub}}^{\mathrm{ac}}<0.05$ with 1032 elements. The adaptive strategy preferentially refines the four corners of the domain, although the refinement is quite uniform as expected. The evolution of the actual error and the bounds is also shown in figure 9. As can be seen, the upper bound $\|e\|_{\mathrm{ub}}^{\mathrm{ac}}$ nearly follows the path of the actual error $\|e\|$ (in the figure they are practically undistinguishable) whereas the equilibrated bounds $\|e\|_{\mathrm{ub}}^{\mathrm{eq}}$ have the same rate of convergence but provide worst effectivities.

\subsection{Linearly forced square domain}

The second example is a linearly forced square domain with the forcing and non-homogeneous boundary conditions chosen to produce the exact solution

$$
u(x, y)=\frac{3}{2} y^{2}(1-y)+2 x y .
$$

The domain is the unit square $\Omega=(0,1) \times(0,1)$ and the boundary conditions are of Dirichlet type on the bottom $y=0$ and Neumann on the remainder of the boundary. Note that both the forcing $f=9 y-3$ and the Neumann boundary conditions are linear functions.

Again the behavior of the bounds is analyzed for a uniform mesh refinement. The initial mesh is composed of 32 triangular elements and in each refinement step every triangle is divided into four similar triangles. Both the initial mesh and the finite element approximation obtained with the mesh of 32768 elements are shown in figure 10.

The upper bounds for the error in the energy norm and their qualities are shown in table IV. Again, it is clear that flux-free strategies provide much better effectivities than the flux equilibration strategies and the minimization of the complementary energy accounting for the accumulated nature of the flux field provides very good effectivity indices. It is worth noting 

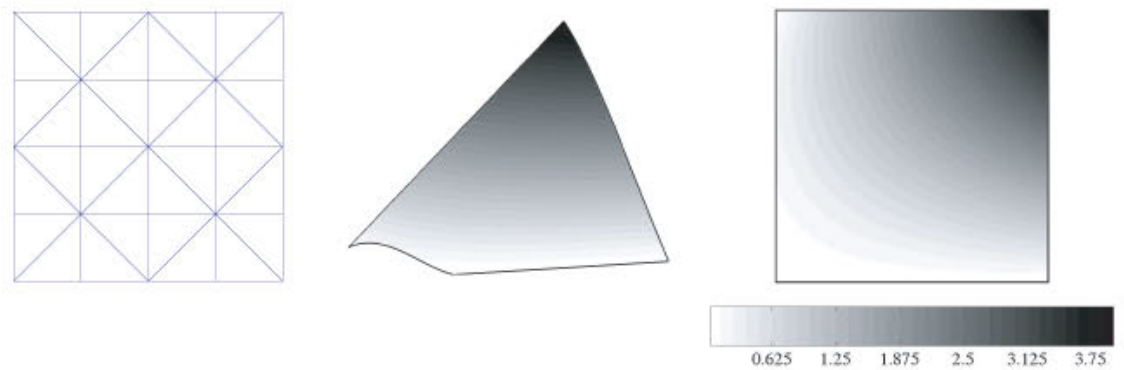

Figure 10. Example 2: series of uniformly $h$-refined linear triangular meshes. Initial mesh (left), finite element approximation on the mesh with 32768 elements (center - 2D view and right - 1D view).

\begin{tabular}{c|cc||ccc||cccc}
\hline \multicolumn{3}{l|}{} & \multicolumn{3}{c||}{ bounds } & \multicolumn{3}{c}{ effectivities } \\
\hline$n_{\mathrm{el}}$ & $\left\|u_{H}\right\|$ & $\|e\|$ & $\|e\|_{\mathrm{ub}}$ & $\|e\|_{\text {ub }}^{\mathrm{ac}}$ & $\|e\|_{\text {ub }}^{\mathrm{eq}}$ & $\|e\| /\left\|u_{H}\right\|$ & $\rho$ & $\rho^{\mathrm{ac}}$ & $\rho^{\mathrm{eq}}$ \\
\hline 32 & 3.25841 & 0.59114 & 0.62625 & 0.59786 & 0.98774 & 0.18142 & $5.94 \%$ & $1.14 \%$ & $67.09 \%$ \\
128 & 3.29778 & 0.30219 & 0.31878 & 0.30502 & 0.50958 & 0.09164 & $5.49 \%$ & $0.93 \%$ & $68.63 \%$ \\
512 & 3.30810 & 0.15213 & 0.16011 & 0.15330 & 0.25660 & 0.04599 & $5.24 \%$ & $0.76 \%$ & $68.67 \%$ \\
2048 & 3.31072 & 0.07622 & 0.08008 & 0.07670 & 0.12839 & 0.02302 & $5.07 \%$ & $0.63 \%$ & $68.44 \%$ \\
8192 & 3.31138 & 0.03813 & 0.04002 & 0.03834 & 0.06416 & 0.01152 & $4.96 \%$ & $0.53 \%$ & $68.24 \%$ \\
32768 & 3.31154 & 0.01907 & 0.02000 & 0.01916 & 0.03206 & 0.00576 & $4.90 \%$ & $0.48 \%$ & $68.12 \%$ \\
\hline
\end{tabular}

Table IV. Example 2: upper bounds for the error in the energy norm in a series of uniformly $h$-refined linear triangular meshes and their quality.

that such a remarkable improvement of the accumulated strategy obtained in the two first examples is quite particular and it is probably due to the underlying regular mesh. Note that the behavior of the accumulated strategy depends not only of the underlying problem but also on the numbering of the elements and for unstructured meshes although it will yield improvements on the effectivities, these improvements will not be such noteworthy, as will be seen in the following examples. Figure 11 shows the spatial distribution of the squared

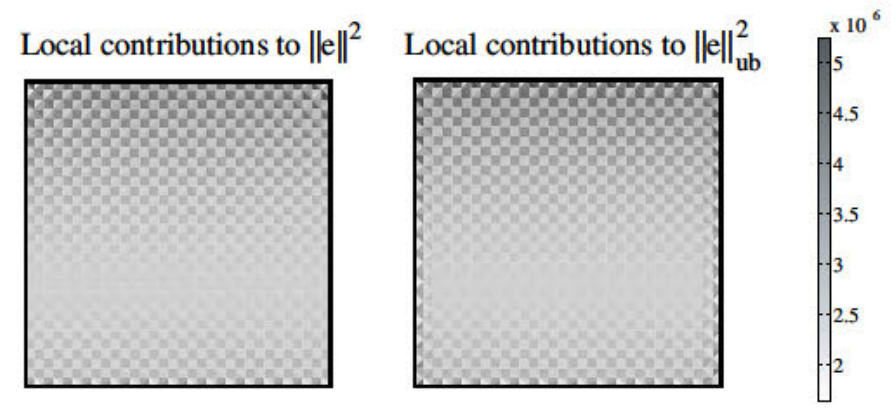

Figure 11. Example 2: spatial distribution of the exact energy norm $\|e\|^{2}$ (left) and of the upper bound $\left(\|e\|_{\mathrm{ub}}^{\mathrm{ac}}\right)^{2}$ (right) for the intermediate mesh of 2048 elements.

energy norm $\|e\|^{2}$ and the spatial distribution of the accumulated flux-free estimate for the intermediate mesh of 2048 elements. As can be seen as in the first example there is a very good agreement between both spatial distribution. The local effectivity indices of the accumulated flux-free estimate, the non-accumulated flux-free estimate and the equilibrated estimate are shown in figure 12. It is clear that the flux-free strategies provide sharper bounds globally and locally. It can also be seen that in this example, the accumulated strategy presents a very good histogram map with most local effectivity indices around 1.

A series of adapted meshes is produced by subdividing at each remeshing step $20 \%$ of the 

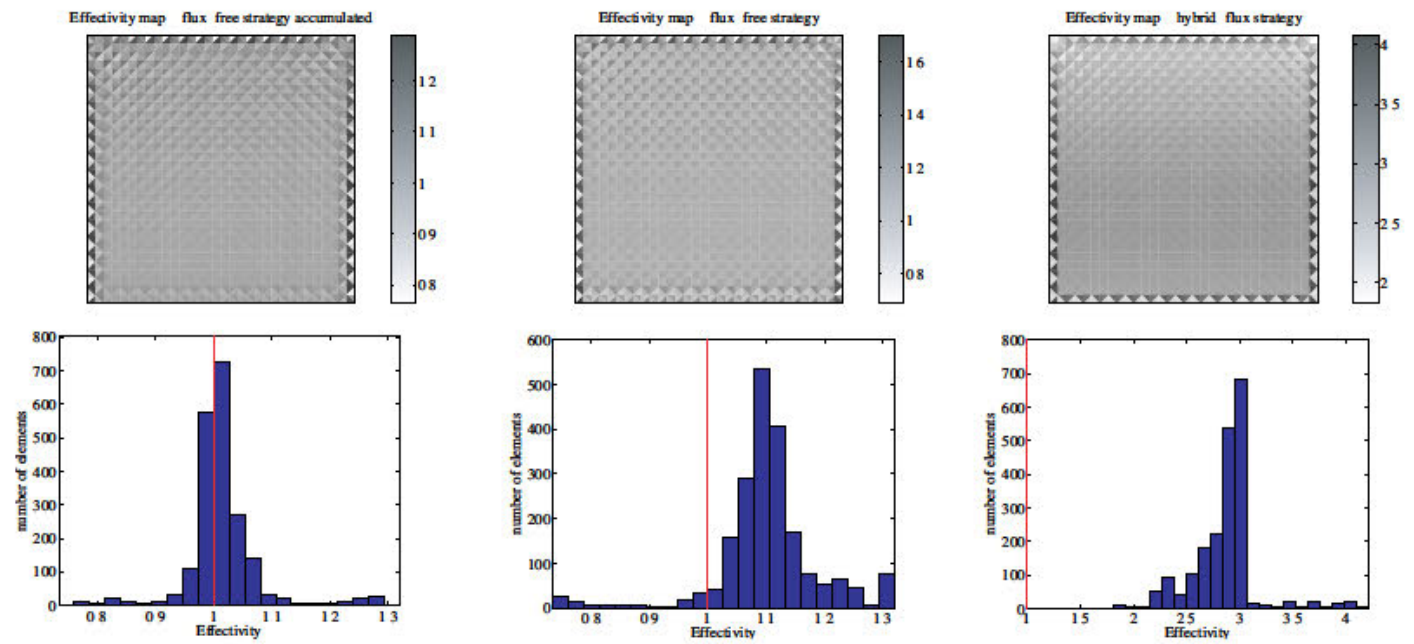

Figure 12. Example 2: spatial distribution of the local effectivity index for the accumulated flux-free technique (left), for the non-accumulated flux-free technique (center) and for the hybrid-flux technique (right). Histograms for local effectivity indices for the previous techniques (down).

elements, those with larger contribution indicators $\eta_{k}^{\text {ac }}$. For this particular problem the error is nearly uniformly distributed, and the adaptive refinement does not produce a decrease in the computational cost as can be seen in figure 13. The convergence of the bounds is the $\mathcal{O}(h)$ both using a uniform or and adaptive refinement. Both in the uniform and the adaptive procedures, the upper bound provided by the accumulated flux-free strategy is in very good agreement with the exact energy norm. Note that in the uniform case it is difficult to distinguish between the exact and the upper bound norms.

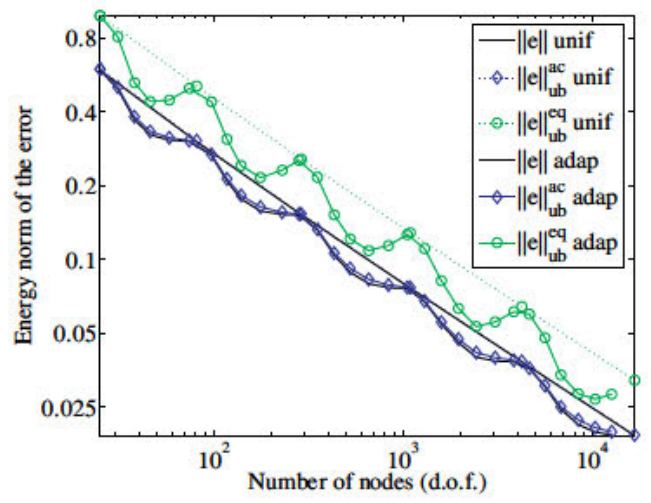

Figure 13. Example 2: Comparison of the convergence of the bounds in a uniform mesh refinement and in the adaptive refinement.

\subsection{The Dirichlet/Neumann discontinuity}

Here we consider the problem in the unit square $\Omega=(0,1) \times(0,1)$ with $\Gamma_{N}=(0,0.5) \times\{1\}$, $\Gamma_{\mathrm{D}}=\partial \Omega \backslash \Gamma_{\mathrm{N}}$. The boundary conditions are homogeneous and $f=1$. This is a standard model problem where the solution exhibits a singularity at the point $(0.5,1)$ due to the change in the type of boundary conditions [32]. The exact solution behaves like $r^{1 / 2} \sin (\theta / 2)$, hence the expected convergence rate is $\mathcal{O}\left(h^{1 / 2}\right)$ in the energy norm if a uniform mesh refinement is used.

The rate of convergence for a uniform mesh refinement may be observed in figure 14 where the upper bounds for the energy norm (for both the accumulated flux-free approach and the equilibrated approach) are plotted in logarithmic scale. The convergence rate is shown in 


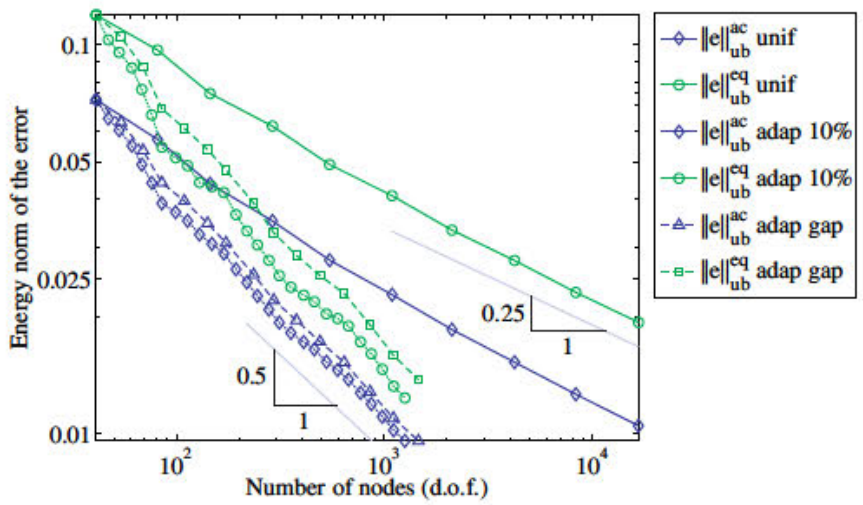

Figure 14. Example 3: Comparison of the convergence of the bounds in a uniform mesh refinement and in the adaptive refinement.

dependence on the total number of unknowns $n_{\text {np }}$, thus, the slope should be $1 / 4$ since $\mathcal{O}\left(h^{1 / 2}\right)$ implies $\mathcal{O}\left(1 / n_{\mathrm{np}}{ }^{1 / 4}\right)$. Also, figure 14 shows that using an adaptive mesh refinement based on the local contributions $\eta_{k}^{\text {ac }}$ drastically improves the convergence rate for the upper bounds. The convergence rate for the adaptive procedures is $\mathcal{O}(h)$ or equivalently $\mathcal{O}\left(1 / n_{\mathrm{np}}{ }^{1 / 2}\right)$. Two different adaptive strategies are used. In the first strategy, at each remeshing step $10 \%$ of the elements are subdivided (those with larger contribution to the square of the upper bound) until the desired tolerance $\|e\|_{\mathrm{ub}}^{\mathrm{ac}}<0.01$ is achieved. Second, at each remeshing step the elements with $\eta_{k}^{\mathrm{ac}}>\left(\|e\|_{\mathrm{ub}}^{\mathrm{ac}}\right)^{2} / n_{\mathrm{el}}$ are subdivided again until the desired tolerance is achieved. As can be seen in figure 14 both strategies yield the same improvement in the convergence rate and provide similar results. Whereas the first strategy needs 28 iterations to converge, the second strategy converges with 15 iterations.
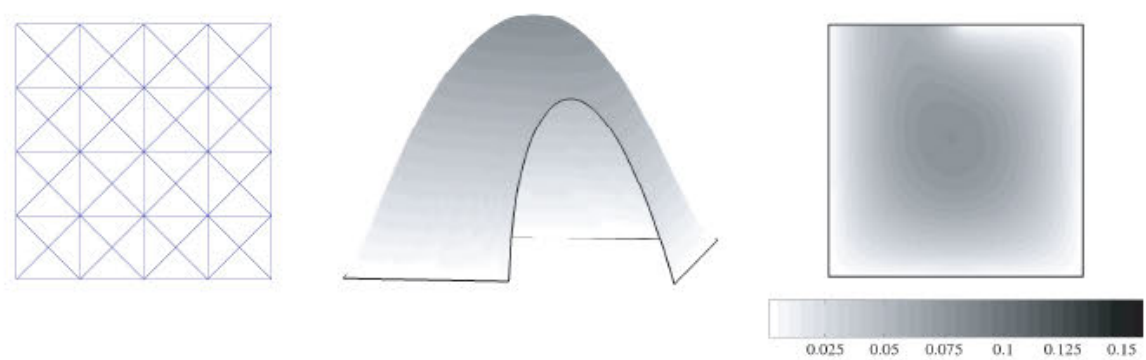

Figure 15. Example 3: series of uniformly $h$-refined linear triangular meshes. Initial mesh (left), finite element approximation on the mesh with 32768 elements (center - 2D view and right - 1D view).

The initial mesh for both the uniform and the adaptive refinements is shown in figure 15 along with the finite element approximation on the final mesh of the uniform refinement. Figure 16 shows the two final meshes of the adaptive procedure with 2359 elements for the strategy where $10 \%$ of the elements are refined in each step and with 2753 elements for the strategy where the elements with $\eta_{k}^{\mathrm{ac}}>\left(\|e\|_{\mathrm{ub}}^{\mathrm{ac}}\right)^{2} / n_{\mathrm{el}}$ are refined. Note that both strategies yield similar final meshes.

The spatial distribution of the upper bound for the accumulated flux-free strategy is shown in figure 17 for three different intermediate meshes. As can be seen, the error is much larger in the elements containing the point where the singularity occurs than on the rest of the elements of the mesh. Also figure 17 shows the elements marked for refinement using the second strategy (refinement of the elements verifying $\left.\eta_{k}^{\mathrm{ac}}>\left(\|e\|_{\mathrm{ub}}^{\mathrm{ac}}\right)^{2} / n_{\mathrm{el}}\right)$. Note that as expected, in all the refinement steps the elements containing the singularity are refined. 

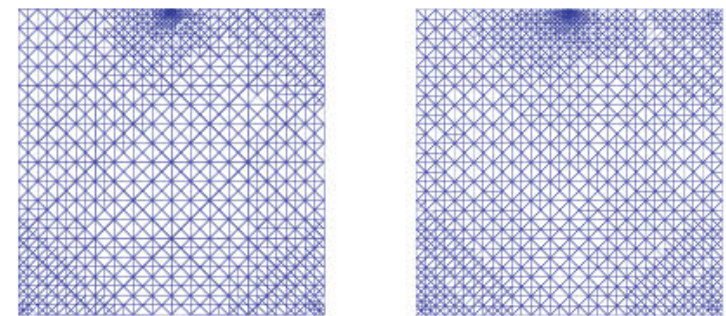

Figure 16. Example 3: series of adaptively $h$-refined linear triangular meshes. Final mesh for the strategy where $10 \%$ of the elements are refined with 2359 elements (left). Final mesh for the strategy where the elements with $\eta_{k}^{\mathrm{ac}}>\left(\|e\|_{\mathrm{ub}}^{\mathrm{ac}}\right)^{2} / n_{\mathrm{el}}$ are refined with 2753 elements (right).
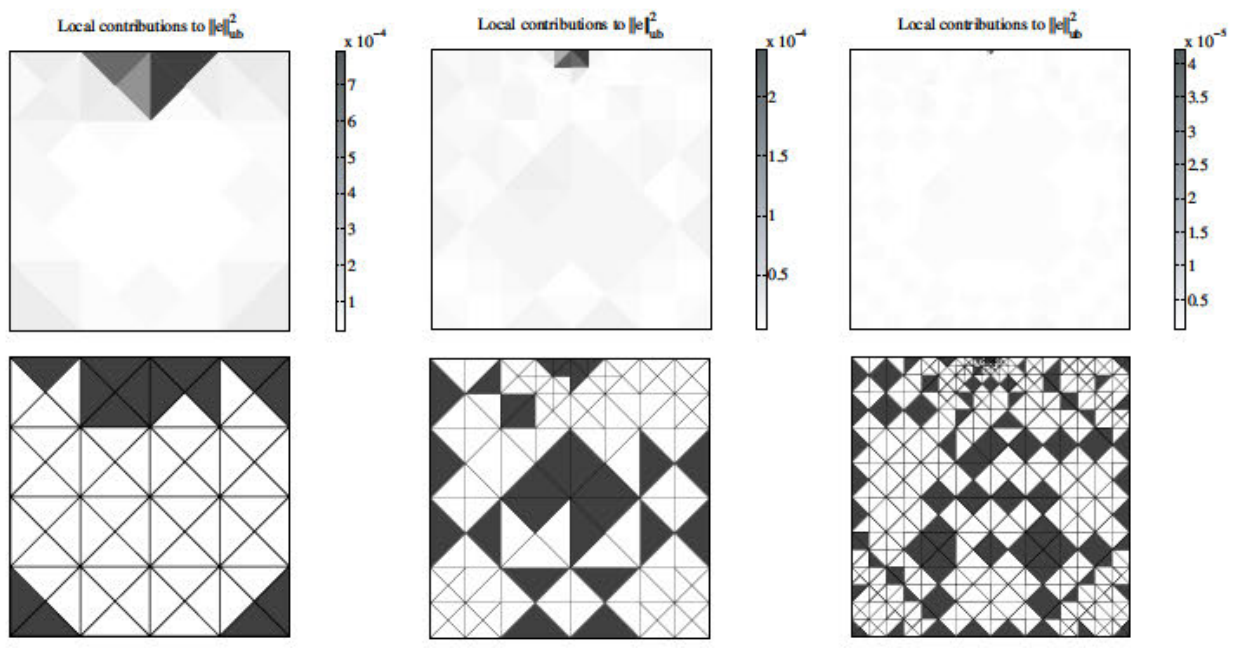

Figure 17. Example 3: series of adaptively $h$-refined linear triangular meshes. Local error distribution $\left(\eta_{k}^{\mathrm{ac}}\right)$ of the accumulated flux-free strategy for the initial and intermediate meshes verifying $\|e\|_{\mathrm{ub}}^{\mathrm{ac}}<$ 0.075 (left), $\|e\|_{\mathrm{ub}}^{\mathrm{ac}}<0.05$ (center) and $\|e\|_{\mathrm{ub}}^{\mathrm{ac}}<0.025$ (right). Below the elements marked for subdivision using the strategy $\eta_{k}^{\mathrm{ac}}>\left(\|e\|_{\mathrm{ub}}^{\mathrm{ac}}\right)^{2} / n_{\mathrm{el}}$ are shown.

\subsection{L-shaped domain with analytic solution}

In this example the Laplace equation is considered in a non-convex domain, $f=0$. The domain is the standard L-shaped domain with a reentrant corner $\Omega=(-1,1) \times(0,1) \cup(-1,0) \times(-1,0)$. The boundary conditions are chosen to produce the exact solution

$$
u(r, \theta)=r^{\frac{2}{3}} \sin \left(\frac{2}{3} \theta\right),
$$

where $r$ is the distance from the reentrant corner point and $\theta$ is the angle from the upper surface of the corner $[0,1] \times\{0\}$. In particular, homogeneous Dirichlet boundary conditions are considered on $\Gamma_{\mathrm{D}}=[0,1] \times\{0\} \cup\{0\} \times[-1,0]$ and on the rest of the boundary, Neumann boundary conditions are applied. The smooth tractions $g$ are computed using the exact solution of the problem.

Since the fluxes on the Neumann boundary $g$ are not piecewise polynomial functions, the strict strategies do not guarantee that the upper bounds are indeed an actual upper bound for the error $\|e\|$. Given a finite element mesh, the Neumann boundary conditions are linearly interpolated and therefore, the bounds are guaranteed for the modified interpolated problem. However, if the Neumann boundaries are carefully refined, the influence of the interpolation error on the Neumann boundary becomes negligible. Even though the bounds are strict with respect to the modified interpolated problem, the results are compared with the exact solution given in (37).

First, the convergence of the bounds is tested using a uniform refinement. For this example, the finite element approximation converges as $\mathcal{O}\left(h^{2 / 3}\right)$ and as can be seen in figure 18 the 
bounds for the energy match the optimal convergence rate. It can be seen that in this case,

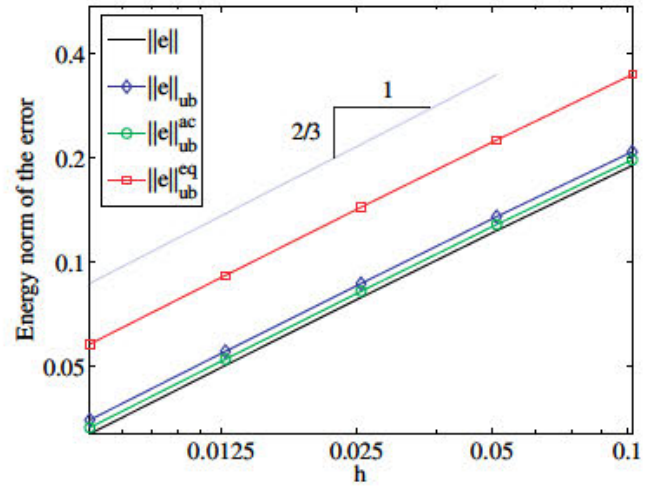

Figure 18. Example 4: series of uniformly $h$-refined linear triangular meshes. Convergence of the bounds.

the influence of the interpolation error on the Neumann boundary is negligible due to the fact that the mesh is uniformly refined (and thus the Neumann boundaries are also refined). This yields bounds which overestimate the exact energy norm for all the strategies. The initial mesh and finite element solution for the final mesh may be seen in figure 19.
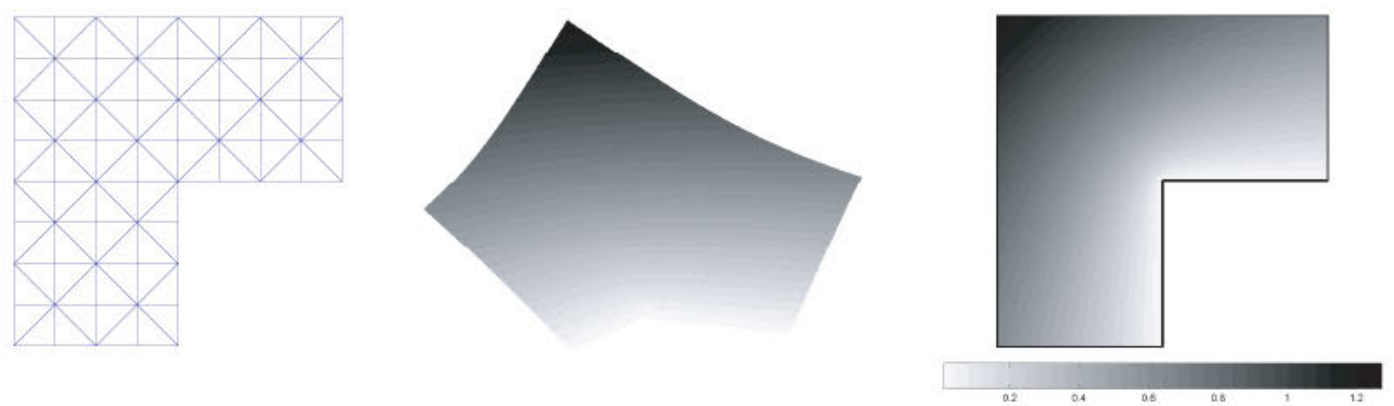

Figure 19. Example 4: series of uniformly $h$-refined linear triangular meshes. Initial mesh with 96 elements (left), finite element approximation on the mesh with 24576 elements (center - 2D view and right - 1D view).
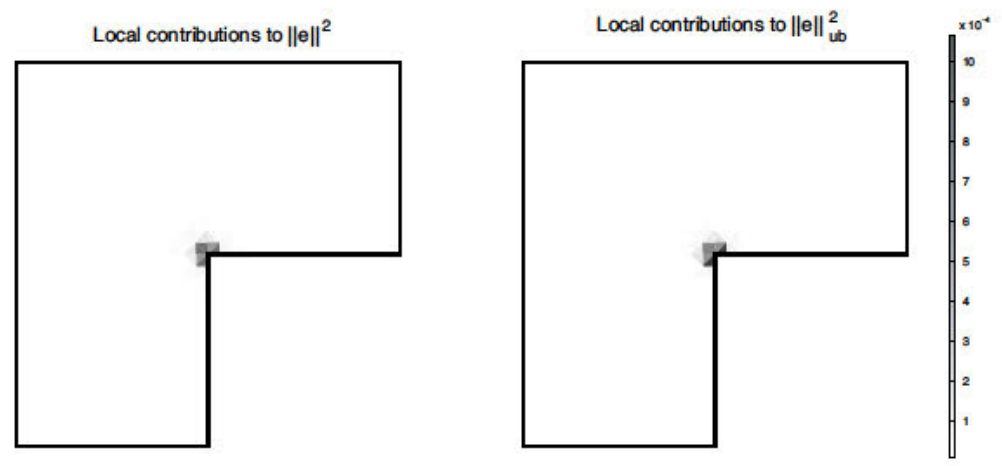

Figure 20. Example 4: spatial distribution of the exact energy norm $\|e\|^{2}$ (left) and of the upper bound $\left(\|e\|_{u \mathrm{~b}}^{\mathrm{ac}}\right)^{2}$ (right) for the intermediate mesh of 1536 elements.

Figure 20 shows the spatial distribution of the squared energy norm $\|e\|^{2}$ and the spatial distribution of the accumulated flux-free estimate for the intermediate mesh of 1536 elements. 

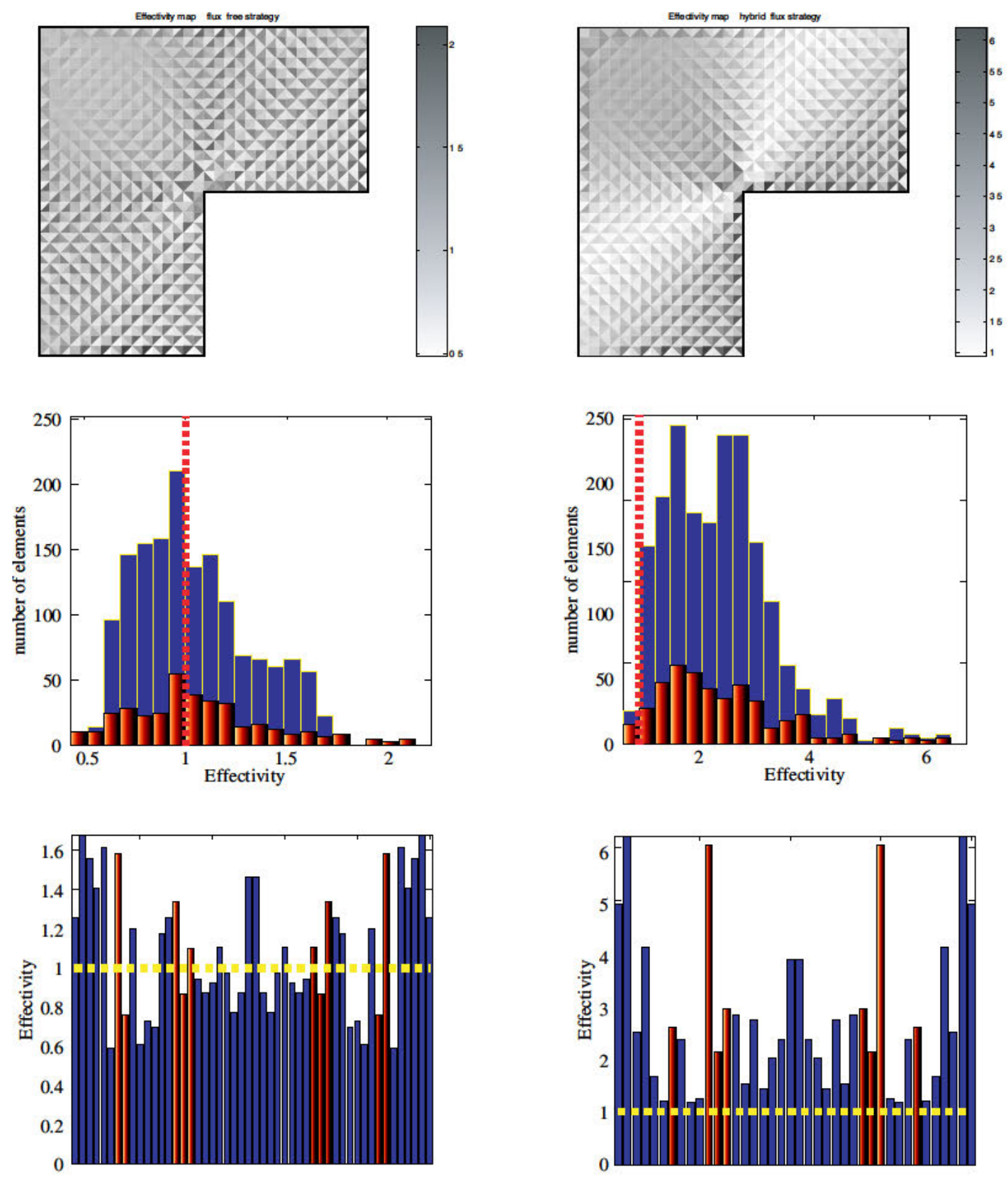

Figure 21. Example 4: spatial distribution of the local effectivity index for the accumulated fluxfree technique (left) and for the hybrid-flux technique (right). In the center the histograms for local effectivity indices for the previous techniques are shown. On the bottom the effectivities of the elements contributing more than $0.1 \%$ to the upper bound are shown.

As can be seen there is a very good agreement between both spatial distribution. Moreover, the elements with larger error are those containing the singularity. The local effectivity indices of the accumulated flux-free estimate and the equilibrated estimate are shown in figure 21 . It is clear that the flux-free strategies provide sharper bounds globally and locally. It can also be seen that in this example, the accumulated strategy presents a very good histogram map with most local effectivity indices around 1 . Moreover, in the histogram the effectivities of the elements lying on a star that intersects the Neumann boundary are highlighted. As can be seen, for the flux-free strategy, the elements with larger effectivity indices are precisely on the 
Neumann boundary. It is worth noting, although, that the contribution of the elements on the Neumann boundary to the exact error is less than $0.0038 \%$ whereas the larger contribution is $23 \%$.

In this example, since the singularity is the main source of error in the energy norm, there are few elements which significantly contribute to the error. Thus, sharp bounds will be obtained if in those selected elements, the local contribution of the upper bound is in good agreement with the exact local contribution. Figure 21 also shows the effectivities of the elements which contribute more than $0.1 \%$ to the upper bound of the error. For the accumulated flux-free strategy only 50 elements contribute more than $0.1 \%$ to the upper bound whereas for the equilibrated strategy there are 40 elements contributing more than $0.1 \%$ of the total bound. The local contribution of the rest of the elements is less than $0.001\|e\|_{\mathrm{ub}}^{2}$. The local effectivities of these elements are shown in figure 21. In these figures the elements contributing more than $1 \%$ to the upper bound have also been highlighted (10 and 8 elements in the flux-free strategy and hybrid-flux strategy respectively). As can be seen, the local effectivities of those elements in the flux-free approach are all near one whereas the hybrid-flux strategy presents a quite large overestimation of the local error.

Finally, since the exact solution is known, one can compute the elements with local contribution to the exact error greater than $0.1 \%$ of the total error $\|e\|^{2}$ ( 60 elements). Figure 22 shows the effectivities of those elements along with the effectivities of the elements contributing more than $1 \%$ of the total error (10 elements). It is worth noting that the contribution of the
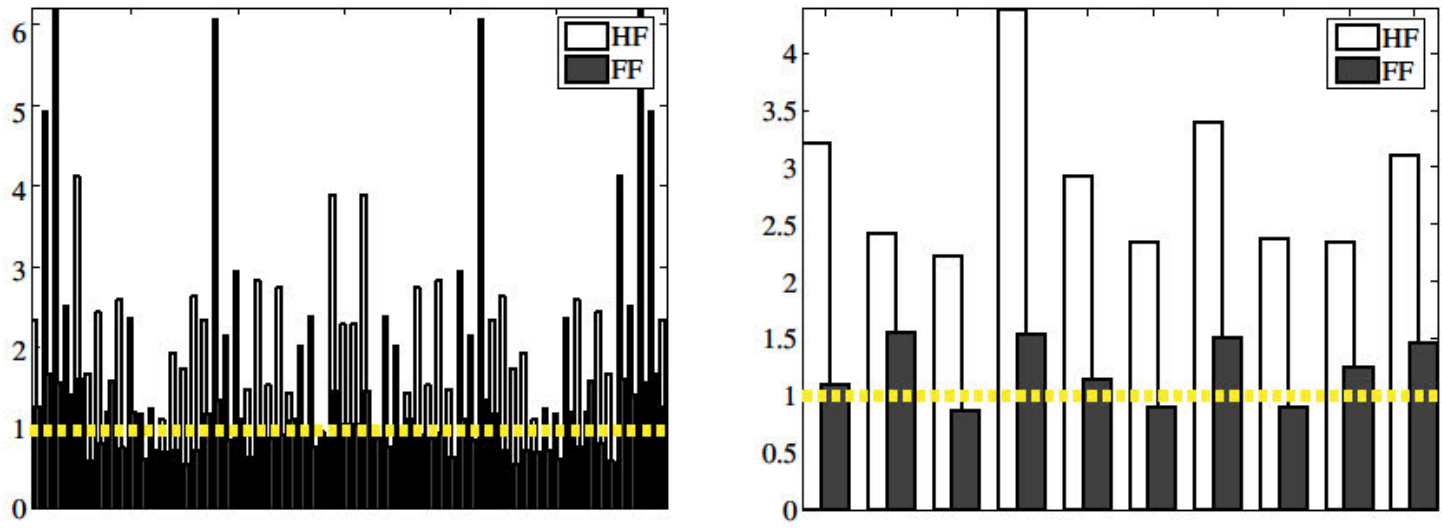

Figure 22. Example 4: effectivities of the estimates for those elements contributing more than $0.1 \%$ (left) and $1 \%$ (right) of the total error.

elements on the Neumann boundary to the local exact error is less than $0.003 \%$ whereas the larger contribution is $22.9 \%$.

The behavior of the bounds is also analyzed using an adaptive refinement. Two different refinement strategies are considered. First, in each remeshing step $30 \%$ of the elements are subdivided (those with larger contribution to the square of the upper bound). Second, in each remeshing step also the $30 \%$ of the elements with larger contribution to the upper bound are refined, but also the Neumann contour is refined in the case that the element size on the Neumann boundaries is larger than either the average element size of the mesh or 50 times the minimum element size. Both refinement strategies are stopped when the desired tolerance $\|e\|_{\mathrm{ub}}^{\mathrm{ac}}<0.01$ is achieved.

For this problem, the convergence of the bounds when a uniform mesh refinement is considered is $\mathcal{O}\left(h^{2 / 3}\right)$ or equivalently $\mathcal{O}\left(n_{\mathrm{np}}{ }^{-1 / 3}\right)$. Adaptive strategies should at least recover the rate $\mathcal{O}(h)=\mathcal{O}\left(n_{\mathrm{np}}{ }^{-1 / 2}\right)$. As can be seen in figure 23 both adaptive strategies achieve the expected convergence rate. In this figure, the bounds obtained using the accumulated fluxfree strategy and the bounds using the hybrid-flux techniques are shown along with the exact energy norm. As can be seen, the hybrid-flux bounds clearly overestimate the exact error.

In all the obtained meshes, the upper bounds both obtained with the flux-free strategy and the hybrid-flux strategy are an upper bound of the exact error. Thus, the error in the Neumann 


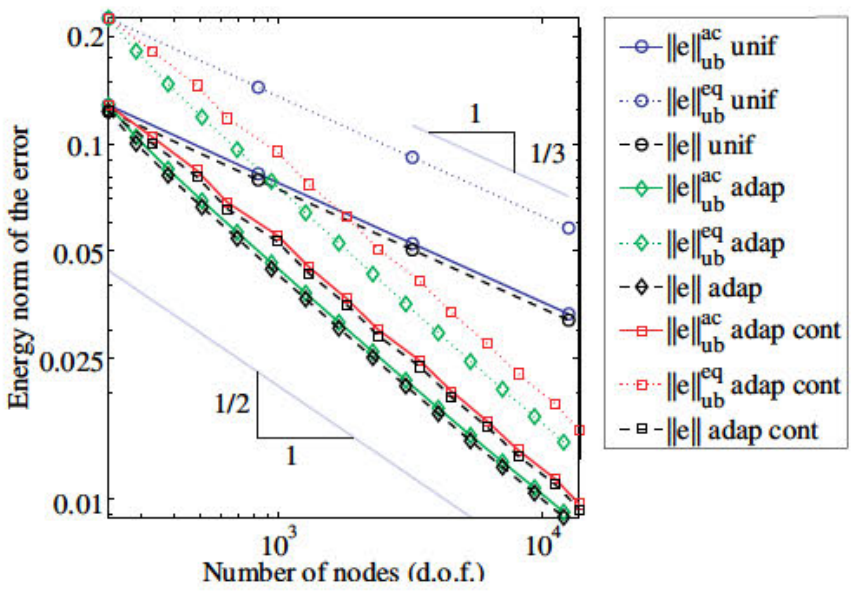

Figure 23. Example 4: comparison of the convergence of the bounds using either a uniform $h$-refinement of using an adaptive $h$-refinement strategy.

boundary conditions does not affect the certification of the bounds, even for the strategy that does not further refine the Neumann boundaries. As mentioned in section 6.4, guaranteed bounds may be obtained by using (only in the stars intersecting the Neumann boundary) the approaches presented in $[17,27]$. However since the error in the Neumann boundary conditions is not affecting the regions of the mesh where the error is larger (due to the singularity), the upper bounds would be nearly the same.
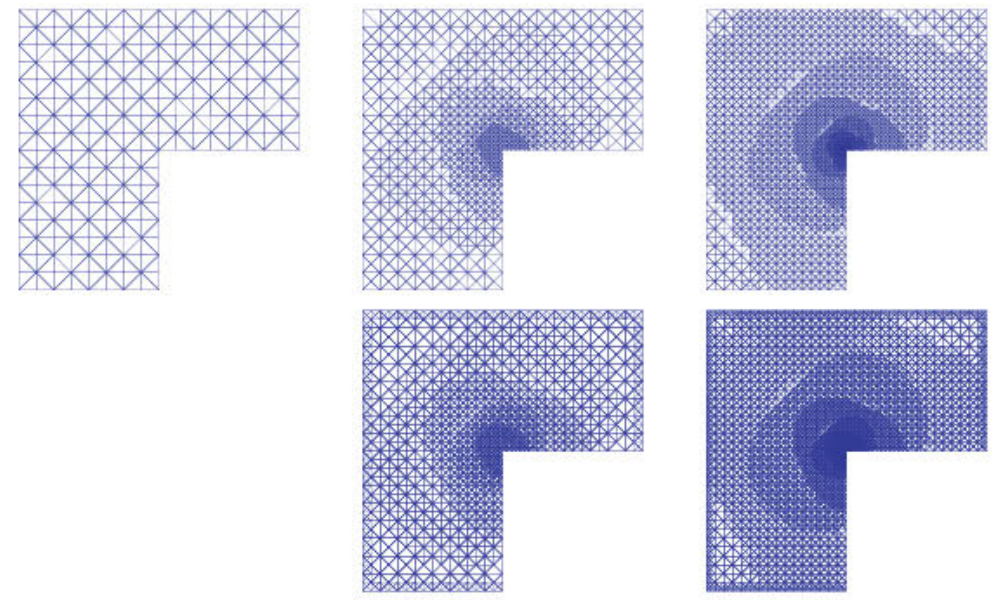

Figure 24. Example 4: initial and intermediate meshes of the adaptive procedure that refine $30 \%$ of the elements in each refinement step (top). Initial and intermediate meshes of the adaptive procedure that refine $30 \%$ of the elements in each refinement step and also refines the Neumann boundaries if necessary (bottom).

The initial and intermediate meshes where $\|e\|_{\text {ub }}^{\text {ac }}<0.05$ and $\|e\|_{\text {ub }}^{\text {ac }}<0.025$ are shown in figure 24 for both adaptive strategies. For the strategy that only uses the information of the local error indicator, the initial and intermediate meshes have $384,1788,5880$ elements respectively. The strategy which also refines the Neumann boundaries starts with the same initial mesh and yields intermediate meshes with 2456 and 6588 elements. As has been seen in figure 23, it is not necessary to refine the Neumann boundaries to yield strict upper bounds for the exact energy norm of the error.

Finally, table V shows the results for the adaptive procedure guided by the local error indicators. Both the accumulated and non-accumulated flux-free bounds are shown for the initial mesh and for the meshes where $\|e\|_{\mathrm{ub}}^{\mathrm{ac}}<0.1,0.05,0.025,0.015$ and 0.01 . As can be 
seen, in this example the accumulated strategy improves the effectivity index by a factor 2 which is not such a remarkable behavior than in the first two examples. The effectivity indices associated with the hybrid-flux strategy are all above $60 \%$.

\begin{tabular}{c|cc||cc||cc}
\hline \multicolumn{3}{l|}{} & \multicolumn{2}{c||}{ bounds } & \multicolumn{2}{c}{ effectivities } \\
\hline$n_{\mathrm{el}}$ & $\left\|u_{H}\right\|$ & $\|e\|$ & $\|e\|_{\mathrm{ub}}$ & $\|e\|_{\mathrm{ub}}^{\mathrm{ac}}$ & $\rho$ & $\rho^{\mathrm{ac}}$ \\
\hline 384 & 1.3492583 & 0.1232893 & 0.1353269 & 0.1283561 & $9.76 \%$ & $4.11 \%$ \\
686 & 1.3524293 & 0.0814326 & 0.0891861 & 0.0847853 & $9.52 \%$ & $4.12 \%$ \\
1788 & 1.3541502 & 0.0444125 & 0.0479465 & 0.0462233 & $7.96 \%$ & $4.08 \%$ \\
5880 & 1.3548864 & 0.0207903 & 0.0223122 & 0.0215768 & $7.32 \%$ & $3.78 \%$ \\
10428 & 1.3549815 & 0.0145790 & 0.0155984 & 0.0151040 & $6.99 \%$ & $3.60 \%$ \\
23662 & 1.3550356 & 0.0088861 & 0.0095018 & 0.0091826 & $6.93 \%$ & $3.34 \%$ \\
\hline
\end{tabular}

Table V. Example 4: upper bounds for the error in the energy norm in a series of adaptively $h$-refined linear triangular meshes and their quality.

\section{CONCLUDING REMARKS}

This paper introduces a new technique to compute strict upper bounds for the energy norm of the error in the finite element approximation in the context of the Poisson equation. The bounds are obtained using a modification of the flux-free technique presented in [18] where asymptotic bounds for the energy norm are computed. The strategy is described in detail emphasizing the numerical implementation of the bound procedure.

The main advantage of the presented flux-free approach with respect to the hybrid-flux methods is that the local problems are self-equilibrated and there is no need to compute the equilibrated flux to ensure the solvability of the local problems. The value of the fluxes of the statically admissible flux field at interelementary boundaries are implicitly computed by the flux-free strategy, and do not require the user to make an initial guess on the value of the fluxes as in the hybrid-flux method (the fluxes on the edges are taken to be linear and an initial constant guess for the fluxes is computed using the finite element approximation). This causes that the flux-free approach yields much sharper bounds than the hybrid-flux method.

It is worth noting that the upper bounds for the energy norm may be used to assess the accuracy of numerical approximations of quantities of interest taking into account that the error in a quantity of interest may be rewritten in terms of energy norms [5].

Finally, the distribution of the local contributions to the error are well suited to guide adaptive procedures.

\section{REFERENCES}

1. Parés N, Díez P, Huerta A. Exact bounds for linear outputs of the advection-diffusion-reaction equation using flux-free error estimates. In preparation. ; .

2. Geradin MG ( (ed.)). B. M. Fraeijs de Veubeke memorial volume of selected papers. Martinus Nijhoff Publishers: The Hague, 1980. Solid Mech. Arch. 1980, Special Memorial Vol.

3. Kelly DW. The self-equilibration of residuals and complementary a posteriori error estimates in the finite element method. Internat. J. Numer. Methods Engrg. 1984; 20(8):1491-1506.

4. Ladevèze P, Leguillon D. Error estimate procedure in the finite element method and applications. SIAM J. Numer. Anal. 1983; 20(3):485-509.

5. Ainsworth M, Oden JT. A posteriori error estimation in finite element analysis. John Wiley \& Sons: Chichester, 2000.

6. Babuška I, Strouboulis T. The finite element method and its reliability. Numerical Mathematics and Scientific Computation, The Clarendon Press Oxford University Press: New York, 2001.

7. Ladevèze P, Pelle JP. Mastering calculations in linear and nonlinear mechanics. Mechanical Engineering Series, Springer-Verlag: New York, 2005. Translated from the 2001 French original by Theofanis Strouboulis.

8. Ladevèze P, Maunder EAW. A general method for recovering equilibrating element tractions. Comput. Methods Appl. Mech. Engrg. 1996; 137(41):111-151. 
9. Maunder EAW, Zhong HG, Beckers P. A posteriori error estimators related to equilibrium defaults of finite element solutions for elastostatic problems. Finite Elem. Anal. Des. 1997; 26(3):171-192.

10. Almeida Pereira OJB, Moitinho de Almeida JP, Maunder EAW. Adaptive methods for hybrid equilibrium finite element models. Comput. Methods Appl. Mech. Engrg. 1999; 176(1-4):19-39. New advances in computational methods (Cachan, 1997).

11. Sauer-Budge AM, Bonet J, Huerta A, Peraire J. Computing bounds for linear functionals of exact weak solutions to Poisson's equation. SIAM J. Numer. Anal. 2004; 42(4):1610-1630 (electronic).

12. Sauer-Budge AM, Peraire J. Computing bounds for linear functionals of exact weak solutions to the advection-diffusion-reaction equation. SIAM J. Sci. Comput. 2004; 26(2):636-652 (electronic).

13. Parés N, Bonet J, Huerta A, Peraire J. The computation of bounds for linear-functional outputs of weak solutions to the two-dimensional elasticity equations. Comput. Methods Appl. Mech. Engrg. 2006; 195(46):406-429.

14. Xuan ZC, Parés N, Peraire J. Computing upper and lower bounds for the $J$-integral in two-dimensional linear elasticity. Comput. Methods Appl. Mech. Engrg. 2006; 195(4-6):430-443.

15. Parés N, Díez P, Huerta A. Bounds of functional outputs for parabolic problems. Part I: Exact bounds of the discontinuous galerkin time discretization. Comput. Methods Appl. Mech. Engrg. 2008; 197(1920):1641-1660.

16. Parés N, Díez P, Huerta A. Bounds of functional outputs for parabolic problems. Part II: Bounds of the exact solution. Comput. Methods Appl. Mech. Engrg. 2008; 197(19-20):1661-1679.

17. Vejchodský T. Guaranteed and locally computable a posteriori error estimate. IMA J. Numer. Anal. 2006; 26(3):525-540.

18. Parés N, Díez P, Huerta A. Subdomain-based flux-free a posteriori error estimators. Comput. Methods Appl. Mech. Engrg. 2006; 195(4-6):297-323.

19. Díez P, Parés N, Huerta A. Recovering lower bounds of the error by postprocessing implicit residual a posteriori error estimates. Internat. J. Numer. Methods Engrg. 2003; 56(10):1465-1488.

20. Cottereau R, Díez P, Huerta A. Strict error bounds for linear solid mechanics problems using a subdomainbased flux-free method. Submitted;

21. Moitinho de Almeida JP, Maunder EAW. A technique for recovery of equilibrium on star patches via a partition of unity. Proceedings of the 5th ECCOMAS 83 8th World Congress on Computational Mechanics, Venice 2008; .

22. Machiels L, Maday Y, Patera AT. A "flux-free" nodal Neumann subproblem approach to output bounds for partial differential equations. C. R. Acad. Sci. Paris Sér. I Math. 2000; 330(3):249-254.

23. Carstensen C, Funken SA. Fully reliable localized error control in the FEM. SIAM J. Sci. Comput. 1999/00; 21(4):1465-1484 (electronic).

24. Morin P, Nochetto R, Siebert K. Local problems on stars: a posteriori error estimators, convergence, and performance. Math. Comp. 2003; 72(243):1067-1097.

25. Sauer-Budge AM, Bonet J, Huerta A, Peraire J. Computing bounds for linear functionals of exact weak solutions to Poisson's equation. SIAM J. Numer. Anal. 2004; 42(4):1610-1630.

26. Parés N, Bonet J, Huerta A, Peraire J. The computation of bounds for linear-functional outputs of weak solutions to the two-dimensional elasticity equations. Comput. Methods Appl. Mech. Eng. 2005; 194.

27. Křížek M. Conforming equilibrium finite element methods for some elliptic plane problems. RAIRO Anal. Numér. 1983; 17(1):35-65.

28. Oden JT, Feng Y. Local and pollution error estimation for finite element approximations of elliptic boundary value problems. J. Comput. Appl. Math. 1996; 74(1-2):245-293. TICAM Symposium (Austin, TX, 1995).

29. Repin S, Sauter S, Smolianski A. A posteriori error estimation for the Poisson equation with mixed Dirichlet/Neumann boundary conditions. Proceedings of the 10th International Congress on Computational and Applied Mathematics (ICCAM-2002), vol. 164/165, 2004; 601-612.

30. Carstensen C, Hoppe RHW. Convergence analysis of an adaptive nonconforming finite element method. Numer. Math. 2006; 103(2):251-266.

31. Paraschivoiu M, Peraire J, Patera AT. A posteriori finite element bounds for linear-functional outputs of elliptic partial differential equations. Comput. Methods Appl. Mech. Engrg. 1997; 150(1-4):289-312. Symposium on Advances in Computational Mechanics, Vol. 2 (Austin, TX, 1997).

32. Repin S, Sauter S, Smolianski A. A posteriori error estimation for the Poisson equation with mixed Dirichlet/Neumann boundary conditions. J. Comput. Appl. Math. 2004; 164/165:601-612. Proceedings of the 10th International Congress on Computational and Applied Mathematics (ICCAM-2002). 